\title{
Pseudomonas aeruginosa Uses c-di-GMP Phosphodiesterases RmcA and MorA To Regulate Biofilm Maintenance
}

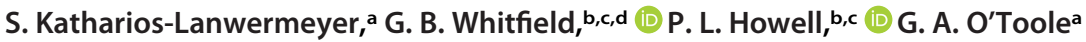 \\ aDepartment of Microbiology and Immunology, Geisel School of Medicine at Dartmouth, Hanover, New Hampshire, USA \\ bProgram in Molecular Medicine, The Hospital for Sick Children, Toronto, Ontario, Canada \\ cDepartment of Biochemistry, University of Toronto, Toronto, Ontario, Canada \\ ¿Département de Microbiologie, Infectiologie et Immunologie, Université de Montréal, Montreal, Quebec, Canada
}

ABSTRACT While the early stages of biofilm formation have been well characterized, less is known about the requirements for Pseudomonas aeruginosa to maintain a mature biofilm. We utilized a $P$. aeruginosa-phage interaction to identify $r m c A$ and morA, two genes which encode bis- $\left(3^{\prime}, 5^{\prime}\right)$-cyclic dimeric GMP (c-di-GMP)-degrading phosphodiesterases (PDEs) and are important for the regulation of biofilm maintenance. Deletion of these genes initially results in an elevated biofilm phenotype characterized by increased production of c-di-GMP, Pel polysaccharide, and/or biofilm biomass. In contrast to the wild-type strain, these mutants were unable to maintain the biofilm when exposed to carbon-limited conditions. The susceptibility to nutrient limitation, as well as subsequent loss of biofilm viability of these mutants, was phenotypically reproduced with a stringent response mutant ( $\Delta$ relA $\Delta s p o T)$, indicating that the $\Delta r m c A$ and $\triangle$ morA mutants may be unable to appropriately respond to nutrient limitation. Genetic and biochemical data indicate that RmcA and MorA physically interact with the Pel biosynthesis machinery, supporting a model whereby unregulated Pel biosynthesis contributes to the death of the $\triangle r m c A$ and $\triangle m o r A$ mutant strains in an established biofilm under nutrient limitation. These findings provide evidence that c-di-GMP-mediated regulation is required for mature biofilms of $P$. aeruginosa to effectively respond to changing availability of nutrients. Furthermore, the PDEs involved in biofilm maintenance are distinct from those required for establishing a biofilm, suggesting that a wide variety of c-di-GMP metabolizing enzymes in organisms such as $P$. aeruginosa allows for discrete control over the formation, maintenance or dispersion of biofilms.

IMPORTANCE Recent advances in our understanding of c-di-GMP signaling have provided key insights into the regulation of biofilms. Despite an improved understanding of how biofilms initially form, the processes that facilitate the long-term maintenance of these multicellular communities remain opaque. We found that $P$. aeruginosa requires two phosphodiesterases, RmcA and MorA, to maintain a mature biofilm and that biofilms lacking these PDEs succumb to nutrient limitation and die. The biofilm maintenance deficiency observed in $\triangle r m c A$ and $\triangle$ morA mutants was also found in the stringent response-defective $\Delta$ relA $\triangle$ spoT strain, suggesting that a regulatory intersection between c-di-GMP signaling, extracellular polysaccharide biosynthesis, and the nutrient limitation response is important for biofilm persistence. We uncover components of an important regulatory system needed for $P$. aeruginosa biofilms to persist in nutrient-poor conditions and provide some of the first evidence that maintaining a mature biofilm is an active process.

KEYWORDS biofilm, c-di-GMP, nutrient limitation, phosphodiesterase, stringent response, Pel polysaccharide

\author{
Citation Katharios-Lanwermeyer S, Whitfield \\ GB, Howell PL, O'Toole GA. 2021. Pseudomonas \\ aeruginosa uses c-di-GMP phosphodiesterases \\ RmcA and MorA to regulate biofilm \\ maintenance. mBio 12:e03384-20. https://doi \\ .org/10.1128/mBio.03384-20. \\ Editor Frederick M. Ausubel, Massachusetts \\ General Hospital \\ Copyright $\odot 2021$ Katharios-Lanwermeyer et \\ al. This is an open-access article distributed \\ under the terms of the Creative Commons \\ Attribution 4.0 International license. \\ Address correspondence to G. A. O'Toole, \\ georgeo@Dartmouth.edu. \\ Received 2 December 2020 \\ Accepted 4 December 2020 \\ Published 2 February 2021
}


seudomonas aeruginosa is a Gram-negative opportunistic pathogen that is found both environmentally and within clinical settings. Able to transition from planktonic lifestyles to a biofilm mode of growth, $P$. aeruginosa biofilms develop via a number of discrete steps generally defined as initial attachment, irreversible attachment, microcolony formation, maturation and dispersal (1). Flagella mediate the initial polar attachment of the cell to the surface, whereas pili facilitate irreversible attachment and commitment to surface growth $(2,3)$. Once on the surface, increased production of extracellular polysaccharide (EPS) facilitates increased surface adhesion and intercellular cohesion and provides both protection and structural integrity required by mature biofilms (3-6).

An important element in the transition of $P$. aeruginosa from a planktonic to the biofilm mode of growth is bis- $\left(3^{\prime}, 5^{\prime}\right)$-cyclic dimeric GMP (c-di-GMP), a second messenger that coordinates the regulatory control of virulence and behaviors needed for surface growth such as motility and the production of EPS (7-10). The concentration of intracellular c-di-GMP is controlled enzymatically by c-di-GMP-synthesizing diguanylate cyclases (DGCs) which contain GGDEF domains, and c-di-GMP degrading phosphodiesterases (PDEs) that harbor EAL or HD-GYP domains $(8,11)$. The genome of $P$. aeruginosa PA14 encodes $\sim 40$ different DGCs and PDEs involved in the regulation of c-di-GMP (12). Despite the rigorous investigations of the contributions of these different enzymes to biofilm formation and dispersion $(13,14)$, our understanding of how they are coordinated and integrated temporally to affect bacterial surface behavior remains incomplete.

Select DGCs and PDEs have been shown to be important during different phases of biofilm formation. For example, the DGC SadC and the PDE BifA both contribute to irreversible attachment and early biofilm formation $(15,16)$. During subsequent biofilm growth, the DGC WspR mediates biofilm maturation by increasing the production of EPS (17). Regulation of c-di-GMP also facilitates the return to the planktonic lifestyle, as evidenced by the PDEs NbdA and DipA, which are required for dispersion in response to changes in levels of nutrients and nitric oxide, respectively $(18,19)$.

While the available literature provides substantial insights into how biofilms form and disperse, our understanding of biofilm maintenance-the process by which existing biofilms regulate themselves to persist on a surface-remains rudimentary. Indeed, it is not even clear if maintenance of the biofilm is an active process. To date, information regarding biofilm maintenance is largely informed by proteomic analysis of biofilms at specific stages of development (20). Such analyses are primarily conducted using biofilms grown under steady-state conditions, leaving open the questions of whether and how the regulation of established biofilms respond to starvation. Here, we provide evidence that the PDEs RmcA and MorA are needed for the maintenance of $P$. aeruginosa biofilms: the loss of either of these PDEs resulted in robust biofilm formation under nutrient-sufficient conditions, but increased cell death and compromised biofilms during starvation.

\section{RESULTS}

CRISPR-activated genetic background reveals role of c-di-GMP signaling in biofilm maintenance. Previously, we reported that the chromosomal integration of a 42-nucleotide sequence of DNA from the bacteriophage DMS3 into the genome of $P$. aeruginosa PA14 resulted in a CRISPR-activated genetic background. The CRISPR-activated strain that carries this 42-nucleotide sequence in the attachment (att) site, called att::DMS3 ${ }_{42}$, was reported to be biofilm-negative due to increased cell death after $24 \mathrm{~h}$, a typical time point to assess biofilm formation in a standard 96-well, static biofilm assay (21). In addition, while the att::DMS3 ${ }_{42}$ strain was able to form a biofilm at early time points $(\sim 6 \mathrm{~h})$ it was largely biofilm-negative by $12 \mathrm{~h}$ (Fig. $1 \mathrm{~A}$, compare the first and fourth bars), suggesting that the biofilm formed but could not be maintained. These data suggested that att::DMS3 ${ }_{42}$ could serve as an effective tool to probe for functions required for biofilm maintenance, a poorly characterized aspect of the biofilm life cycle. Our goal then was to exploit the robust phenotype offered by the CRISPR-activated strain to further elucidate the process of biofilm maintenance. 

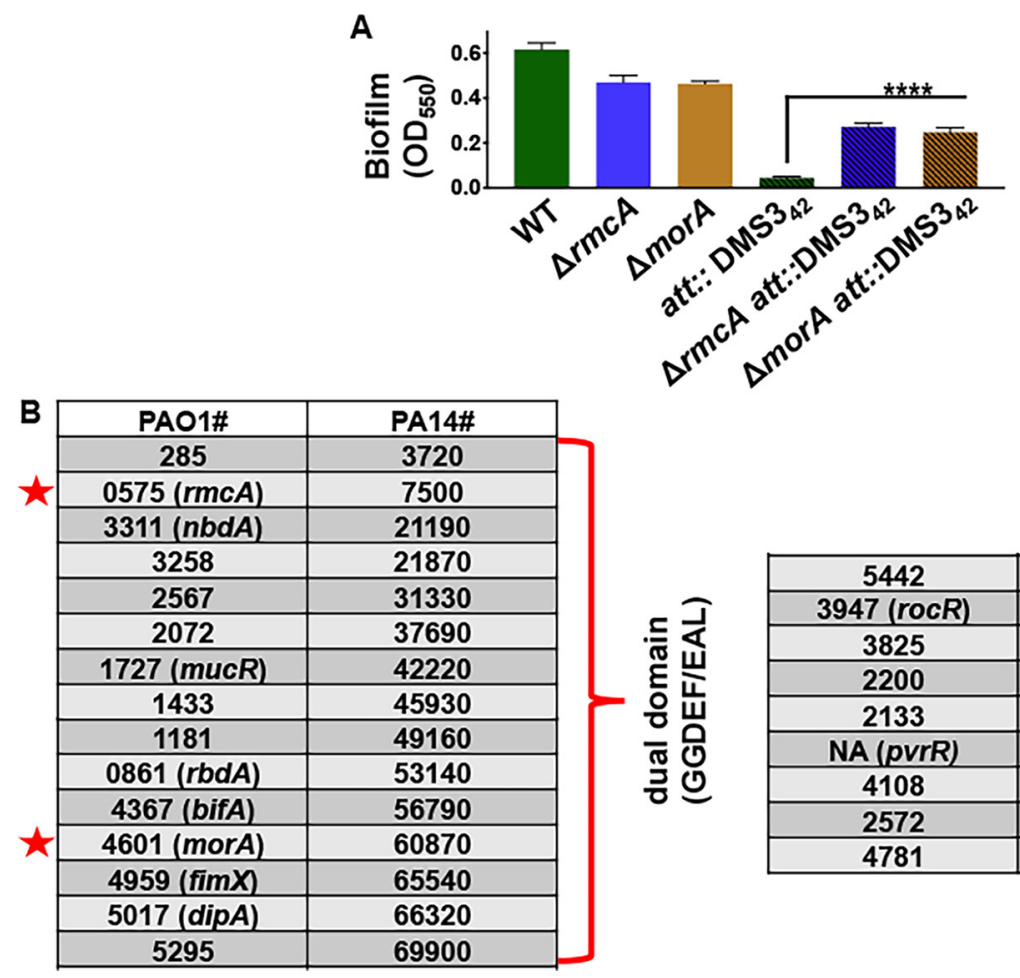

\begin{tabular}{|c|c|}
\hline 5442 & 71850 \\
\hline $3947($ rocR) & 12810 \\
\hline 3825 & 14530 \\
\hline 2200 & 36260 \\
\hline 2133 & 36990 \\
\hline NA (pvrR) & 59790 \\
\hline 4108 & 10820 \\
\hline 2572 & 30830 \\
\hline 4781 & 63210 \\
\hline
\end{tabular}

FIG 1 CRISPR-activated $P$. aeruginosa is unable to maintain a biofilm but can be rescued by introducing mutations in selected PDEs. (A) The $\triangle r m c A$ and $\triangle$ morA mutations in the WT ( $P$. aeruginosa PA14) or the CRISPR-activated background (att::DMS3 ${ }_{42}$ ) were assessed for biofilm formation at $12 \mathrm{~h}$. The $\mathrm{OD}_{550}$ value represents a measure of the biofilm formed. Error bars represent standard deviations of the results from three biological replicates each performed with three technical replicates. ${ }^{* * *}$, a difference in

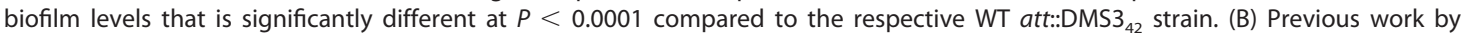
$\mathrm{Ha}$ et al. (23) created in-frame, unmarked deletions of all putative phosphodiesterases that were identified via SMART analyses of proteins encoded in the $P$. aeruginosa genome. The deletion constructs were introduced into the att::DMS3 ${ }_{42}$ background, and mutations in two genes indicated by red stars—rmcA $\left(\Delta r m c A\right.$ att::DMS3 $\left.{ }_{42}\right)$ and $\Delta$ morA $\left(\Delta m o r A\right.$ att::DMS3 $\left.{ }_{42}\right)$ - were found to produce significantly more biofilm compared to the att::DMS3 42 strain, as shown in panel A.

Due to the known role of high levels of c-di-GMP in facilitating biofilm growth (9), we built on the work of $\mathrm{Ha}$ et al., who used the SMART algorithm to analyze $P$. aeruginosa proteins with motifs related to c-di-GMP degradation $(22,23)$. This approach identified 24 candidate PDEs with either EAL domains alone (6), dual GGDEF/EAL domains (15), or HD-GYP domains (3) (Fig. 1B). Individual in-frame deletions for all these PDEs were constructed in the att::DMS3 ${ }_{42}$ background, and these strains were then assayed for biofilm formation. Of all the deletions of genes coding for a PDE that were constructed, only two of them, the $\triangle r m c A$ and $\triangle$ morA mutants, exhibited a significant rescue of biofilm biomass at $12 \mathrm{~h}$ compared to the parental att::DMS3 ${ }_{42}$ background (Fig. 1A, rightmost three bars). While both rmcA and morA encode dual domain (GGDEF and EAL) proteins, previous work suggests that these proteins behave predominately as PDEs $(24,25)$. The observation that none of the other PDEs tested (Fig. 1B) could rescue the biofilm formation defect of the att::DMS3 ${ }_{42}$ strain suggests a specific role for these particular PDEs, a point we address further below.

$\Delta r m c A$ or $\Delta$ morA mutants are defective in the later stages of biofilm formation in a static assay. We next investigated the biofilm phenotypes of the $\triangle r m c A$ and $\triangle$ morA mutants in a wild-type (WT) background (e.g., no $\mathrm{DMS}_{42}$-mediated CRISPR activation) using a 96-well dish static biofilm assay over a $48-\mathrm{h}$ window. These mutants were able to form a biofilm similar to the WT over the first $\sim 12 \mathrm{~h}$ of the assay (Fig. 2A). Between 12 and $24 \mathrm{~h}$, while WT biofilm biomass continued to increase, the biomass of the $\Delta r m c A$ and $\triangle$ morA mutants plateaued at $\sim 24 \mathrm{~h}$ and subsequently decreased through the final time point. Between 24 and $48 \mathrm{~h}$, the WT biofilm is maintained with no obvious loss of biomass in this assay. 
A

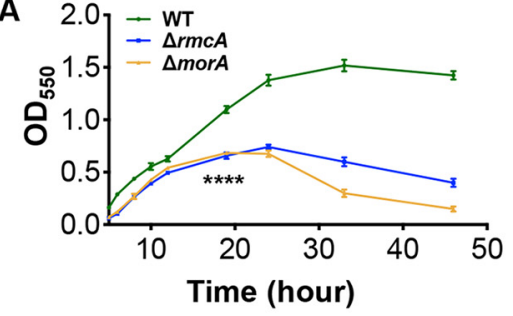

B

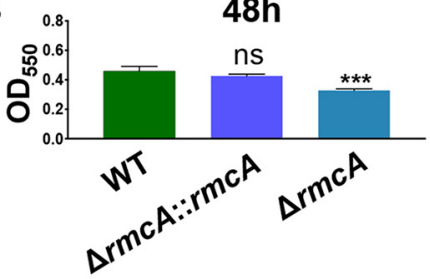

C

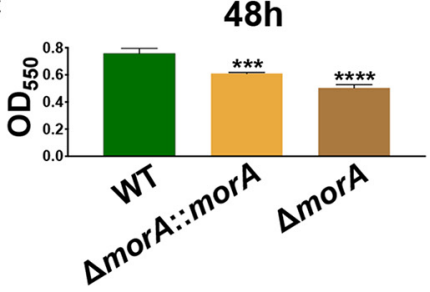

D

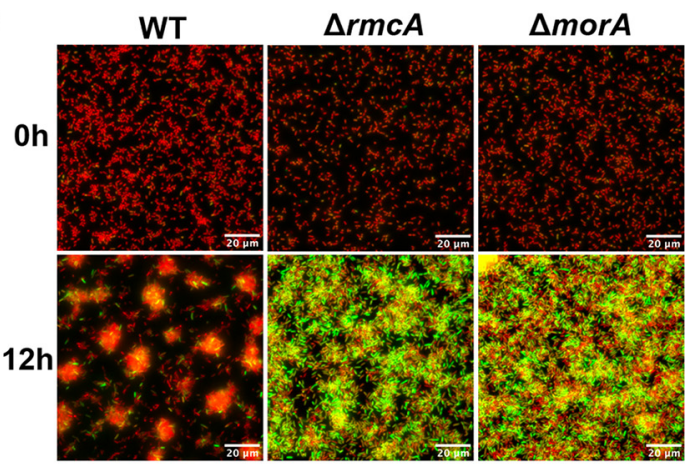

E

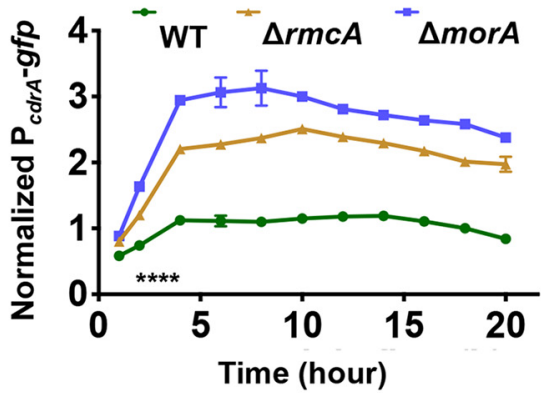

FIG 2 The $\Delta r m c A$ and $\Delta$ morA mutants are defective in biofilm maintenance and show increased levels of c-diGMP. (A) The WT strain ( $P$. aeruginosa PA14) and the $\Delta r m c A$ and $\Delta m o r A$ mutant strains were grown statically in a 96-well dish assay using M63 medium supplemented with $0.4 \%$ L-arginine, and the biofilm was measured at the indicated time points. ${ }^{* * * *}$, a difference in biofilm that is significantly different at $P<0.001$ at the indicated time point and each subsequent time point. (B) The WT, $\Delta r m c A$ mutant, and the $\Delta r m c A:: r m c A^{+}$complemented strain were grown for $48 \mathrm{~h}$ prior to crystal violet staining to assess the extent of biofilm formation. ${ }^{* * *}$, a difference that is significantly different at $P<0.01$ compared to the WT (ns, not significant). (C) The WT, $\Delta$ morA mutant, and the $\Delta$ morA::morA ${ }^{+}$complemented strain were grown for $48 \mathrm{~h}$ prior to crystal violet staining to assess the extent of biofilm formation. ${ }^{* * *}$ and ${ }^{* * *}$, differences that are significantly different at $P<$ 0.01 and $P<0.001$, respectively, compared to the WT. (D) The WT and the $\triangle r m c A$ and $\triangle$ morA mutants carrying the $\mathrm{P}_{c d r A}-g f p$ fusion expressed from a multicopy plasmid were grown in glass bottom 8-well dishes and imaged over $20 \mathrm{~h}$, with a representative image from the $12 \mathrm{~h}$ time point shown. The green color is due to GFP expression, and the orange color is contributed by the constitutively expressed mKO fluorescent protein. (E) Quantification of GFP signal intensity for the strains described in panel D. Fluorescent microscopy was used to determine GFP signal intensity as a measure of c-di-GMP production, which was normalized to constitutively expressed $\mathrm{mKO}$ fluorescent protein. ${ }^{* * * *}$, a difference in signal that is significantly different at $P<0.0001$ compared to the WT strain from 2 to $20 \mathrm{~h}$ of the assay. In all panels, error bars represent standard deviations from three biological replicates each performed with three technical replicates.

To assess whether the behavior of the $\Delta r m c A$ and $\Delta$ morA mutant strains is dependent on the type of carbon source provided, we replaced the carbon source used in our standard medium, L-arginine, with pyruvate. We found that that the $\Delta r m c A$ and $\Delta m o r A$ mutant biofilms also exhibit a late-stage defect at $36 \mathrm{~h}$ in this pyruvate-containing medium, while the 12- $\mathrm{h}$ biofilms were similar to WT (see Fig. S1 in the supplemental material).

To verify that the loss of biofilm that we observed in late time points of our kinetic assay was dependent on the absence of RmcA or MorA, we complemented these mutants and found that the late-stage biofilm defect observed in these mutants could be rescued by a WT copy of $r m c A$ and to a lesser extent for morA (Fig. 2B and C, respectively).

Finally, we wanted to extend our studies to a second strain of $P$. aeruginosa; therefore, we tested $P$. aeruginosa PAO1 $\triangle r m c A$ and $\triangle m o r A$ mutants in biofilm assays and showed, as was the case for these mutants in the PA14 strain background, that the $P$. aeruginosa PAO1 $\triangle r m c A$ and $\triangle$ morA mutants could form early biofilms but had a biofilm defect at later time points (see Fig. S2). 
The $\triangle r m c A$ and $\triangle$ morA mutants have increased c-di-GMP levels. Given that RmcA and MorA are predicted PDEs $(24,25)$, we would expect that the $\triangle r m c A$ and $\triangle$ morA mutants would have increased levels of c-di-GMP compared to the WT strain. To test this idea, biofilms of the WT, $\Delta r m c A$, and $\Delta$ morA strains were grown in glassbottom dishes, and the c-di-GMP-responsive $\mathrm{P}_{c d r A}$-gfp promoter fusion was used to assess transcriptional activity of $c d r A$ over $20 \mathrm{~h}$, with the green fluorescent protein (GFP) signal normalized to constitutively expressed fluorescent protein mKO.

Deletion of either the $r m c A$ or morA gene increased $\mathrm{P}_{c d r A}$-gfp fluorescence (Fig. 2D and $E$ ), as indicated by the enhanced green color, suggesting increased levels of c-diGMP in these two mutants. The structures of the WT and mutant biofilms also differed, with the WT generating typical "mushroom-like" colonies, while the mutants formed a more uniform layer of cells. Thus, it was important to examine the normalized signal intensity of individual cells to accurately compare the average signal intensity between strains. On a cell-by-cell basis, with values normalized to mKO signal intensity, both the $\Delta r m c A$ and $\triangle$ morA mutants exhibited a significant increase in signal intensity from the $\mathrm{P}_{c d r A}$-gfp reporter of 2.8- and 2.1-fold over WT, respectively (Fig. 2E). These results indicate that both rmcA and morA encode active PDEs, as reported $(24,25)$ and that the absence of these enzymes results in reduced degradation of c-di-GMP and thus higher levels of this second messenger.

Given the observation that strains with a late-stage biofilm deficiency also had elevated c-di-GMP, we hypothesized that this deficiency could be induced in WT or enhanced in $\triangle r m c A$ and $\Delta$ morA mutants simply by further increasing c-di-GMP production. To test this hypothesis, we measured biofilm levels at several time points in WT and mutant backgrounds that were heterologously expressing gcbC-R363E from a plasmid; the GcbC-R363E mutant protein has a defective I-site that results in overproduction of c-di-GMP. As exhibited in Fig. S3, however, increasing c-di-GMP production alone is insufficient to induce a late-stage biofilm deficiency in the WT, nor is it capable of accelerating the kinetics of the observed biofilm defect in the $\triangle r m c A$ and $\triangle$ morA mutant backgrounds. These data suggest that the observed phenotypes are linked to the loss of rmcA and morA specifically, rather than to a general increase in c-di-GMP levels.

The inability of $\Delta r m c A$ and $\Delta$ morA to maintain a late-stage biofilm is correlated with nutrient limitation. The defect in late-stage biofilms in the static assay suggested that, as nutrients become limited in batch cultures, the $\triangle r m c A$ and $\triangle m o r A$ mutants may be unable to adapt to nutrient depletion, with ensuing loss of viability. To test this idea, we conducted static 96-well biofilm assays in which biofilm formation was measured early ( $16 \mathrm{~h}$ ) and late (48 $\mathrm{h}$ ) and then compared these results to biofilms grown for the same $48 \mathrm{~h}$, but with periodic (every $12 \mathrm{~h}$ ) removal of spent medium followed by the addition of fresh medium. That is, periodic replacement of the medium provided cells with regular access to fresh media even in the batch culture system. We found that, compared to late time points in the standard assay (no medium replacement) wherein the $\Delta r m c A$ and $\triangle$ morA mutants exhibited a pronounced biofilm defect (55.7 and $82.5 \%$ reduction, respectively; see Fig. S4A), the removal and replacement of spent medium with fresh medium results in the reduction of the magnitude of this defect to $11.6 \%$ in $\Delta r m c A$ and $33.1 \%$ in $\Delta m o r A$, respectively (see Fig. S4B). Early-stage biofilms were measured at $12 \mathrm{~h}$ as an additional control (see Fig. S4C).

We next assessed whether oxidative stress could result in a biofilm maintenance defect as what was observed for nutrient limitation. We grew biofilms for $12 \mathrm{~h}$ in a static 96-well assay, a time point wherein we have detected minimal evidence of starvation responses within the biofilm (Fig. 2A; see also Fig. S5A). We next replaced the spent medium with fresh medium supplemented either with $20 \mathrm{mM} \mathrm{H}_{2} \mathrm{O}_{2}$ (see Fig. S5B) or no $\mathrm{H}_{2} \mathrm{O}_{2}$ (see Fig. S5C) and then incubated the biofilms for an additional 6 h. The addition of $\mathrm{H}_{2} \mathrm{O}_{2}$ had no impact on the WT or mutant biofilms (see Fig. S5B) compared to the control (see Fig. S5C). Taken together, these results are consistent with the hypothesis that the defect that we observe in the $\Delta r m c A$ and $\Delta m o r A$ mutants 
A

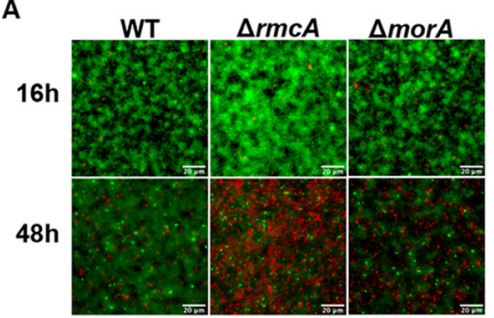

B

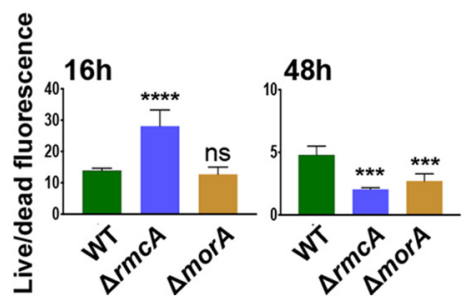

C

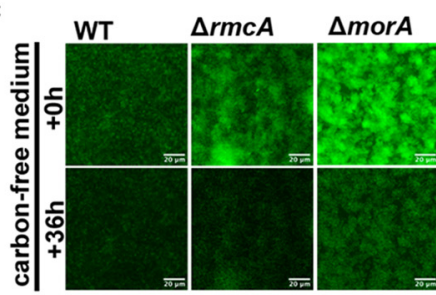

D

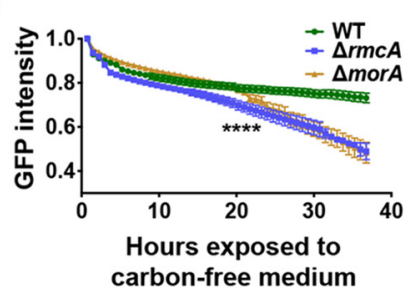

E

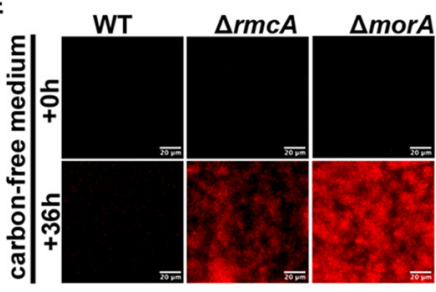

F

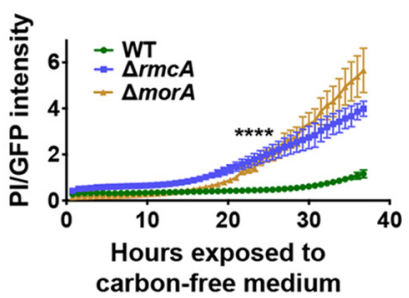

FIG 3 The biofilm defect of $\triangle r m c A$ and $\triangle$ morA mutants coincides with cell death. The WT strain ( $P$. aeruginosa PA14) and the $\triangle r m c A$ and $\triangle$ morA mutants were grown along the air-liquid interface of $18-\mathrm{mm}$ glass coverslips. At $24 \mathrm{~h}$ the coverslips were removed, washed in PBS, and stained with Syto9 and PI. Fluorescent microscopy was used to measure the Syto9 and PI fluorescence $(A)$ and image intensity assessed as a ratio live (Syto9) to dead (PI) was determined at $16 \mathrm{~h}(\mathrm{~B}$, left) and $48 \mathrm{~h}(\mathrm{~B}$, right). Error bars represent standard deviations from three biological replicates each performed with three technical replicates. ${ }^{* *}$ and ${ }^{* * *}$, differences in signal that are significantly different at $P<0.001$ and $P<0.0001$, respectively, compared to the WT; ns, not significant. (C) The WT strain and the $\triangle r m c A$ and $\triangle$ morA mutants carrying plasmid pSMC21 were grown for $23 \mathrm{~h}$ in biofilm medium containing $0.4 \%$ L-arginine and stained under flow in a microfluidic device for an additional $1 \mathrm{~h}$ in biofilm medium containing PI. After this $24-\mathrm{h}$ period of incubation, the biofilm medium was replaced with medium containing PI and lacking arginine; this was considered to be time $=0 \mathrm{~h}$ of nutrient limitation (top panel). The biofilms imaged after $36 \mathrm{~h}$ of nutrient limitation are shown at the bottom of the panel. (D) GFP fluorescence of the biofilms in panel $C$ were measured every 45 min for $36 \mathrm{~h}$. WT and the mutants were assessed for changes in GFP fluorescence and plotted as a fraction of GFP signal relative to the start of nutrient limitation. (E) PI staining of the corresponding biofilms from panel $C$ is shown just prior to and after 36 h of nutrient limitation. (F) PI straining normalized to GFP fluorescence of pSMC21 is plotted for data acquired in panels C and E every $45 \mathrm{~min}$. Error bars represent standard deviation from three biological replicates each performed with three technical replicates. $* * * *$, a difference in biofilm that is significantly different at $P<0.0001$, a level of significance observed after $23 \mathrm{~h}$ of exposure to nutrient-limited conditions, and at all subsequent time points.

is due to an inability to appropriately respond to nutrient limitation, rather than a general stress response.

The inability of the $\Delta r m c A$ and $\triangle m o r A$ mutants to maintain an established biofilm correlates with increased cell death. To explain the loss of late-stage biofilms in the $\Delta r m c A$ and $\triangle m o r A$ mutants, we tested the hypothesis that these mutants were dying, thereby causing the loss of biofilm biomass. We imaged biofilms after staining with a LIVE/DEAD BacLight kit (Molecular Probes). This assay allowed us to compare viability at each time point by determining the ratio of cells that are viable (i.e., cells stained green by membrane-permeable Syto9) to those that are dead (i.e., cells with compromised membranes that are stained red by membrane-impermeable propidium iodide). Biofilms were stained with BacLight after $16 \mathrm{~h}$ or $48 \mathrm{~h}$ of static growth in 12well plates, as described above, and the data plotted as the ratio of live cells (green) to dead cells (red).

After $16 \mathrm{~h}$, biofilms of all strain backgrounds were comprised of predominantly viable cells (Fig. 3A, top row) with the $\triangle$ morA mutant displaying a live/dead ratio similar to the WT, while the $\Delta r m c A$ mutant live/dead ratio was significantly higher than the WT even at $16 \mathrm{~h}$ due largely to the increased biomass of this mutant (Fig. 3B, left panel). After $48 \mathrm{~h}$ in the static assay, however, both the $\Delta r m c A$ and the $\Delta$ morA mutants exhibited decreased viability, (Fig. $3 \mathrm{~A}$, bottom row) with the live/dead ratios of $\Delta r m c A$ and $\triangle$ morA mutants reduced by 55.7 and $43.3 \%$, respectively, in comparison to WT at this late time point (Fig. 3B, right panel). While both mutants exhibited a reduced ratio of live/dead cells, individual comparisons of Syto9 and PI (see Fig. S6A and B, respectively) reveal that the reduction observed in the $\Delta r m c A$ mutant is driven both by an increase in the number of dead cells and a decrease in live cells, whereas for the $\Delta m o r A$ mutant the change in this ratio reflects primarily the loss of viable (green) cells. 
The inability to detect a significant increase in dead cells within late-stage $\Delta$ morA biofilm could be due to an earlier onset of death, followed by the sloughing off of dead cells prior to microscopy at $48 \mathrm{~h}$, a conclusion consistent with the findings presented below.

To formally test that the late-stage biofilm defect of $\Delta r m c A$ and $\Delta m o r A$ mutations that we observed in static conditions was due to nutrient limitation, we utilized a microfluidic device that allowed us to observe biofilm dynamics in real time and to manipulate the amount of nutrients provided. We first confirmed that all three strains could form a biofilm in a microfluidic chamber. To monitor biofilm formation, we introduced the pSMC21 plasmid, which constitutively expresses $\operatorname{GFP}(26,27)$, into each of the strains. The bacteria were inoculated into the microfluidic chamber, allowed to attach for $1 \mathrm{~h}$ prior to the start of flow $(0.5 \mu \mathrm{l} / \mathrm{min})$, and then monitored at $45-\mathrm{min}$ intervals using fluorescence microscopy over the first $12 \mathrm{~h}$ of biofilm formation. The $\Delta r m c A$ and $\triangle$ morA mutants are able to form biofilms in the microfluidic chamber, with the $\Delta r m c A$ mutant exceeding the WT and no significant difference observed between the WT and $\Delta$ morA mutant strains (see Fig. S7).

We hypothesized that we could recapitulate in a microfluidic chamber the nutrientlimited conditions that developed over late time points in the static assays by establishing the biofilms in a medium that contained a carbon source and then irrigating the biofilms with medium lacking a carbon source. To test this idea, we allowed WT and mutant strains carrying the GFP-expressing plasmid pSMC21 to form a biofilm for $24 \mathrm{~h}$ in biofilm medium with arginine as the carbon source and then switched to biofilm medium lacking arginine. At $1 \mathrm{~h}$ before the switch to nutrient-limited conditions, we stained the microfluidic chamber-grown biofilms with propidium iodide ( $\mathrm{PI}$ ) to label nonviable cells and thus establish a baseline of nonviable cells before inducing nutrient limitation. As shown in Fig. $3 C$ and $E$, at time zero before nutrient limitation is induced, all three strains were able to form a biofilm and showed minimal nonviable cells.

To assess whether nutrient limitation differentially impacted the biofilm of the WT compared to the mutants, we normalized the GFP signal to the start of nutrient limitation for each strain ( $t=0 \mathrm{~h}$ in Fig. 3C, top) and then recorded the change in GFP intensity over the subsequent $36 \mathrm{~h}$ of exposure to carbon-free medium. Although the WT was largely able to maintain the biofilm over the course of the assay, the lack of carbon in the growth medium accelerated the loss of biomass in both $\Delta r m c A$ and $\triangle m o r A$ mutant biofilms. This conclusion was supported by the significantly greater reduction of the GFP-mediated signal intensity in the mutants compared to the WT during the 36-h period of carbon limitation (Fig. 3C and D). The WT lost $\sim 25 \%$ of biomass compared to a $>50 \%$ reduction for both the $\Delta r m c A$ and the $\Delta m o r A$ mutants over the $36 \mathrm{~h}$ of the experiment.

To assess whether these mutants lost biofilm biomass over the course of nutrient limitation due to increased death, as observed in the static assay in Fig. 3A, we measured the intensity of PI over time and found that both the $\triangle r m c A$ and $\triangle$ morA mutants exhibited significantly increased PI staining in absolute terms compared to the WT (Fig. 3E) and as a ratio of GFP fluorescence intensity, which served as a measure of total biofilm biomass of viable cells (Fig. 3F). These data provide further evidence that both the $\Delta r m c A$ and $\triangle m o r A$ mutants are susceptible to nutrient-limited conditions when grown in a biofilm and that loss of late-stage biofilm biomass coincides with cell death.

To determine whether dispersal of the biofilm could contribute to the observed loss of biofilm biomass upon nutrient limitation, we measured the viable count (CFU) of bacteria dispersing from biofilms grown in a microfluidic device prior to and after nutrient limitation. As shown in Fig. S8, effluent-derived cells of all strains were similar $2 \mathrm{~h}$ prior to, as well 12 and $18 \mathrm{~h}$ after, the switch to arginine-free medium. Only in the final time point $(24 \mathrm{~h}$ ) did the viable count of the $\Delta r m c A$ and $\triangle m o r A$ effluent increase compared to the WT. These data suggest that cell death, and not dispersal, is the primary driver of the loss of biomass observed in these PDE mutants during nutrient 
A

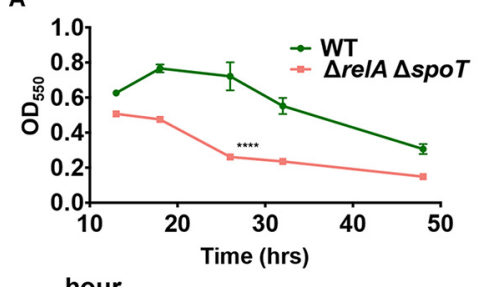
hour

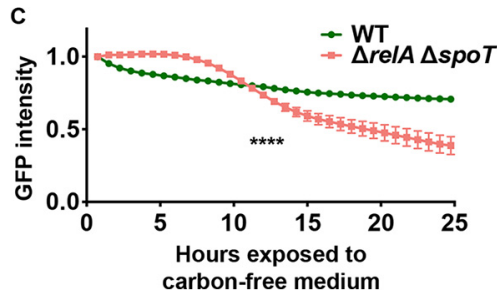

B
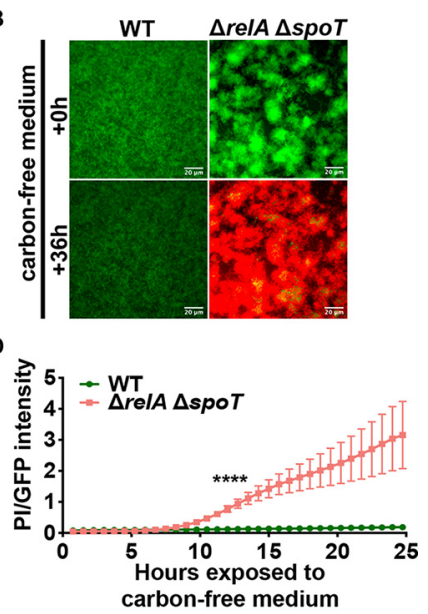

FIG 4 Loss of stringent response phenocopies biofilm defect and cell death observed for the PDE mutants. (A) The WT ( $P$. aeruginosa PA14) strain and the $\Delta$ relA $\Delta$ spoT double mutant strain were grown statically in a 96-well dish assay using M63 medium supplemented with $0.4 \%$ L-arginine, and biofilm formed was measured at the indicated time points. ${ }^{* * *}$, a difference in biofilm that is significantly different at $P<0.0001$ at the indicated time point and the subsequent time point. (B) The WT strain and the $\Delta$ relA $\Delta$ spoT double mutant, both carrying pSMC21, were grown for $23 \mathrm{~h}$ using a microfluidic device in biofilm medium containing $0.4 \%$ L-arginine and stained under flow for an additional $1 \mathrm{~h}$ in biofilm medium containing PI. The biofilm medium was then replaced with medium containing PI and lacking arginine. The fluorescence due to GFP and PI for the WT strain and the $\Delta$ relA $\Delta$ spoT double mutant are shown at the start of nutrient limitation ( $0 \mathrm{~h}$, top) and after $36 \mathrm{~h}$ (bottom) in the microfluidic chamber. (C) GFP fluorescence was measured every 45 min for $24 \mathrm{~h}$ after the initiation of nutrient limitation. The WT strain and the $\Delta$ relA $\Delta$ spoT double mutant strain were assessed for changes in GFP fluorescence, which was normalized to the GFP signal at the start of nutrient limitation, which is set to 1 . (D) The ratio of PI to GFP during $\sim 25 \mathrm{~h}$ of nutrient limitation in the microfluidic chamber is presented as a measure of cell viability, with larger values indicating more cell death. For all panels, error bars represent standard deviations from three biological replicates each performed with three technical replicates. Results from a representative experiment are shown. ${ }^{* * *}, a$ difference in biofilm that is significantly different at $P<0.0001$ at the indicated time point and at each subsequent time point.

limitation, although the loss of cells does contribute to the observed phenotypes at late time points.

A stringent response mutant phenocopies the biofilm cell death of $\Delta r m c A$ and $\Delta$ morA mutants during nutrient limitation. The ability of the $\triangle r m c A$ and $\triangle m o r A$ mutants to maintain a biofilm when nutrients were present coupled with the decrease in viability during nutrient limitation suggested that these mutants were unable to mediate the appropriate responses needed for persistence when resources become limiting. This conclusion was further supported by the observation that the biofilm defect in the static assay for the $\Delta r m c A$ and $\Delta m o r A$ mutants could be rescued simply by adding fresh medium (see Fig. S4). Based on these data, we hypothesized that loss of RmcA or MorA function results in the inability to appropriately navigate nutrient-limited conditions.

If this hypothesis is correct, other strains defective in the nutrient limitation response should have a similar phenotype. To test this prediction, we assessed a $\Delta$ relA $\Delta$ spoT double mutant, which is unable to either make or degrade (p)ppGpp, the alarmone critical for the stringent response, for its ability to respond to nutrient limitation when grown in a biofilm. We first assessed biofilm formation of the $\Delta$ relA $\Delta$ spoT mutant in static assays and found that, like the $\Delta r m c A$ and $\Delta$ morA mutants (Fig. 2A), the $\Delta$ relA $\Delta$ spoT mutant could form a biofilm (albeit at a level lower than the WT) at early time points, but the biofilm biomass was reduced at later time points (Fig. 4A).

The $\Delta$ relA $\Delta$ spoT mutant was also assessed in a microfluidic chamber where nutrient-limited conditions could be induced via introduction of carbon-free medium. Using constitutive GFP fluorescence and PI staining, we found that $\Delta$ relA $\Delta$ spoT grew robust biofilms when provided sufficient nutrients (Fig. 4B, top panel). However, when 
A

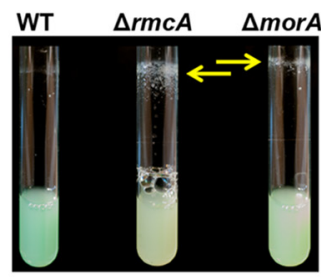

B

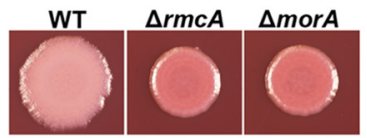

C

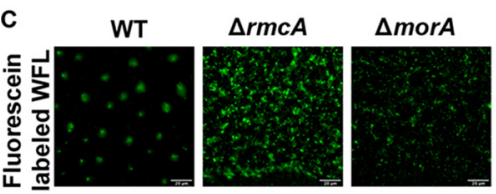

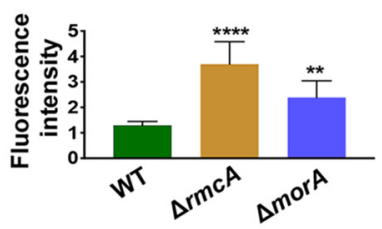

E

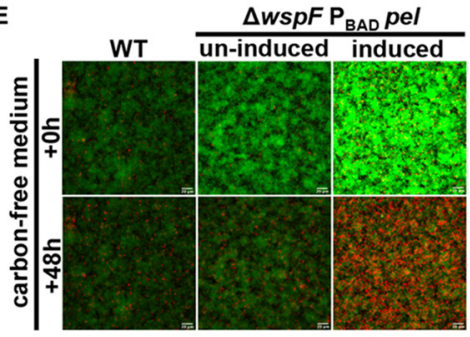

$\mathbf{F}$

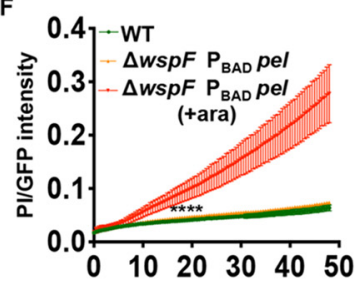

G

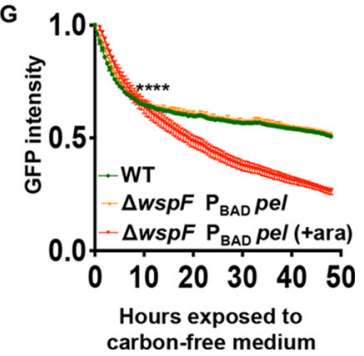

FIG 5 Induction of Pel phenocopies the $\Delta r m c A$ and $\Delta$ morA mutants and is detrimental to flow cell-grown biofilms during starvation. (A) Cultures of the indicated P. aeruginosa PA14 strains were inoculated into lysogeny broth (LB) and imaged after overnight growth at $37^{\circ} \mathrm{C}$. The resulting wall-associated material is indicated by the yellow arrows. (B) Congo red plate assays of the indicated $P$. aeruginosa PA14 strains are shown. The plates were incubated for $24 \mathrm{~h}$ at $37^{\circ} \mathrm{C}$ then at room temperature for an additional 4 days. (C) The WT ( $P$. aeruginosa PA14) strain and the $\Delta r m c A$ and $\Delta m o r A$ mutants were grown as a biofilm in a static assay for $18 \mathrm{~h}$ in medium containing fluorescein-labeled WGA and washed before imaging by fluorescence microscopy. (D) Quantification of the experiment performed in panel C. The fluorescence attributable to the fluorescein-labeled WGA was normalized to the mKO fluorescent protein expressed from the chromosome of each strain and plotted. (E to G) The WT ( $P$. aeruginosa PA14) strain and the $\Delta w s p F P_{B A D}-p e l$ strain, which contained a constitutively expressed GFP introduced in single copy at the att site, were grown for $23 \mathrm{~h}$ using a microfluidic device with biofilm medium containing $0.4 \%$ L-arginine, and then PI was added to the medium to stain dead cells before starvation. $0.2 \%$ arabinose was added to induce $\mathrm{P}_{\mathrm{BAD}}$-pel expression and is indicated by the label "induced" or "+ara." (E) After the biofilm was allowed to establish for $24 \mathrm{~h}$, the biofilm medium was replaced with medium containing PI but lacking arginine (i.e., starvation conditions), and biofilms were imaged over an additional $48 \mathrm{~h}$. The top panel shows the biofilm at $24 \mathrm{~h}$ of growth before starvation, and the bottom panel shows the biofilms after $48 \mathrm{~h}$ of nutrient limitation. (F) Viability was assayed as a ratio of PI staining and GFP fluorescence over $48 \mathrm{~h}$ of exposure to nutrient-limited medium. The higher value indicates more cell death. (G) Loss of biofilm was quantified by normalizing GFP's fluorescence intensity to the signal quantified immediately after switching to nutrient-limited medium. For all panels, error bars represent standard deviations from three biological replicates, each performed with three technical replicates. Results from a representative experiment are shown. ${ }^{* \star *}$, a difference in biofilm that is significantly different at $P<0.0001$ at the indicated time point and each subsequent time point.

nutrients were limited the $\Delta$ relA $\Delta$ spoT strain exhibited decreased biomass and enhanced PI staining (Fig. 4B, bottom panel). We then quantified changes in GFP fluorescence and $\mathrm{PI}$ staining over time, we found that the loss of biofilm biomass (Fig. 4C) and viability (Fig. 4D) occurred simultaneously after $\sim 12 \mathrm{~h}$ of exposure to nutrient-limited conditions. Thus, the $\Delta r e l A \Delta s p o T$ mutant displayed phenotypes similar to the $\Delta r m c A$ and $\Delta$ morA mutants when grown as biofilms subjected to nutrient limitation.

RmcA and MorA function is associated with increased Pel polysaccharide production in the biofilm. Increased c-di-GMP is typically associated with enhanced biofilm formation. To reconcile how mutants that have a late-stage biofilm defect (Fig. 3) also produce increased c-di-GMP (Fig. 2D and E), we hypothesized that the loss of the biofilm observed in the $\triangle r m c A$ and $\triangle$ morA mutants was the result of untimely cellular investment in energetically expensive products. Such a view is consistent with the kinetics of the defect observed for the $\Delta r m c A$ and $\Delta$ morA mutants, which becomes increasingly evident after $\sim 30 \mathrm{~h}$ of growth in static conditions, when nutrients are likely depleted, and after a shift to carbon-free medium in the microfluidic device.

To evaluate whether the increased concentration of c-di-GMP in late-stage biofilms also resulted in altered phenotypes relevant to biofilm formation, we assessed production of extracellular polysaccharide (EPS) in the WT and the $\triangle r m c A$ and $\triangle m o r A$ mutants. In P. aeruginosa PA14 the dinucleotide c-di-GMP upregulates Pel production. Both mutants showed enhanced pellicle production compared to the WT, with accumulated biomass on the tubes of overnight-grown planktonic cultures (Fig. 5A). We 
also employed Congo red (CR), a dye which can be used as a qualitative indicator of the presence of EPS, combined with colony biofilm assays on agar medium. The $\Delta r m c A$ and $\triangle$ morA mutants showed enhanced CR binding compared to the WT (Fig. 5B), consistent with the view that the loss of RmcA and MorA results in increased c-di-GMP and EPS production.

To test whether the Pel polysaccharide specifically was being overproduced in the $\Delta r m c A$ and $\triangle m o r A$ mutants, we used fluorescein-labeled Wisteria floribunda lectin (WFL), which binds preferentially to carbohydrate structures that terminate in $\mathrm{N}$-acetylgalactosamine. This lectin has been shown to bind specifically to the Pel polysaccharide (28). Biofilms were grown in a static assay on coverslips that were partially submerged in buffered biofilm medium containing $0.4 \%$ arginine in a 12 -well dish, as described previously (29). To quantify the presence of Pel, we stained statically grown biofilms with WFL and normalized the fluorescent signal to the constitutively expressed fluorescent tag mKO. Biofilms of the $\triangle r m c A$ and $\triangle m o r A$ mutants demonstrated elevated WFL binding (Fig. $5 \mathrm{C}$ ), with signals from the $\triangle r m c A$ and $\triangle m o r A$ mutants significantly increased by 2.8 - and 1.9-fold above the WT, respectively (Fig. 5D). Combined, these data suggest that both $\triangle r m c A$ and $\triangle m o r A$ mutants produce increased levels of the Pel EPS when growing as a biofilm, consistent with the elevated levels of c-di-GMP observed in these mutants (Fig. 2).

Induction of pel when c-di-GMP is overproduced phenocopies the $\Delta r m c A$ and $\Delta$ morA mutants. We next probed whether high levels of Pel expression in the $\Delta r m c A$ and $\triangle$ morA mutants was necessary and/or sufficient to induce late cell death. First, we introduced a $\triangle$ pelA mutation into the $\triangle r m c A$ and $\triangle$ morA backgrounds, but unfortunately, the $\Delta r m c A \Delta$ pelA and $\triangle$ morA $\triangle$ pelA double mutants were defective for establishing a biofilm (see Fig. S9A), which is expected given the role of Pel in biofilm formation (30), so we could not perform the desired analyses in these strains. Instead, we used a strain that allowed us to artificially induce Pel transcription to high levels; this strain did not show a late-stage biofilm defect (see Fig. S9B).

We next hypothesized that induction of a biofilm maintenance defect due to nutrient limiting conditions required a combination of enhanced Pel transcription and elevated c-di-GMP, as demonstrated previously (5). To test this hypothesis, we introduced the arabinose-inducible $\mathrm{P}_{\mathrm{BAD}}$-pel construct, which allows us to induce expression of the pel genes, into the $\Delta w s p F$ mutant background, which has elevated c-di-GMP (5). We then assessed the ability of this strain to endure nutrient limitation in a microfluidic chamber. Compared to the WT, the $\Delta w s p F \mathrm{P}_{\mathrm{BAD}}-$ pel construct produced an increased amount of biofilm compared to the WT over $24 \mathrm{~h}$ (Fig. 5E, compare the top left panel to the top center panel), a phenotype that was further enhanced through the addition of arabinose, which stimulates the transcription of $\mathrm{P}_{\mathrm{BAD}}-$ pel (Fig. 5E, compare the top center panel to the top right panel). These findings are consistent with the important role that both c-di-GMP and Pel play in biofilm development.

After $48 \mathrm{~h}$ of being exposed to a nutrient-limited medium containing PI, the WT and the noninduced $\Delta w s p F \mathrm{P}_{\mathrm{BAD}}-p e l$ were similarly viable, while induced $\Delta w s p F \mathrm{P}_{\mathrm{BAD}^{-}}$ pel exhibited increased PI staining, indicating increased cell death (Fig. 5E, compare the bottom center panel to the bottom right panel). To quantify this loss of viability over time, we measured PI staining as a ratio to GFP, finding that arabinose induced $\Delta w s p F \mathrm{P}_{\mathrm{BAD}}-p e l$ strain exhibited a significant increase in its $\mathrm{PI}$ /GFP ratio over time compared to the WT or noninduced $\Delta w s p F \mathrm{P}_{\mathrm{BAD}}-$ pel (Fig. 5F). Finally, to assess whether induction of pel caused a decrease in biomass, we normalized GFP fluorescence to the start of starvation and assessed the fluorescence intensity over time, finding that only the arabinose induced $\Delta w s p F \mathrm{P}_{\mathrm{BAD}}$-pel strain exhibited a significant decrease in GFP signal (Fig. 5G).

The observation that only the arabinose-induced $\Delta w s p F \mathrm{P}_{\mathrm{BAD}}-p e l$ strain (i.e., enhanced pel transcription and increased c-di-GMP level) exhibited a simultaneous decrease in biomass and enhanced cell death suggests that increased production of Pel is deleterious to $P$. aeruginosa in nutrient-limited conditions. The absence of significant changes in viability or biomass in noninduced $\Delta w s p F P_{B A D}-p e l$ suggests that 
defects in biofilm maintenance observed in induced $\Delta w s p F \mathrm{P}_{\mathrm{BAD}}$-pel biofilms is primarily driven by EPS production and not enhanced levels of c-di-GMP alone.

RmcA and MorA physically interact with the Pel biosynthetic machinery. The data presented thus far suggests that appropriate Pel regulation is lost when cells lack RmcA or MorA function. The biosynthesis of Pel is regulated at the transcriptional and posttranslational levels by the c-di-GMP-binding effector proteins FleQ and PelD, respectively $(31,32)$. DGCs and PDEs can influence the activation state of effector proteins through the alteration of global intracellular c-di-GMP pools or via specific interaction with effectors via local signaling events (33). RmcA, MorA, and PelD localize to the inner membrane due to the presence of one or more predicted transmembrane helices $(24,34,35)$; therefore, we hypothesized that RmcA and/or MorA may influence Pel biosynthesis through direct interactions with PelD. Given that we saw similar latestage biofilms in P. aeruginosa PA14 and PAO1, we took advantage of the available reagents in $P$. aeruginosa $\mathrm{PAO} 1$ to test this hypothesis.

To assess possible RmcA/MorA/PelD interactions, a vesicular stomatitis virus glycoprotein (VSV-G) tag was added to the $C$ terminus of RmcA and MorA (RmcA-V and MorA-V), and the genes expressing these tagged proteins were integrated at the neutral chromosomal att::Tn7 site under the control of the $\mathrm{P}_{\mathrm{BAD}}$-araC promoter in $P$. aeruginosa strains lacking a native copy of the $r m c A$ or morA genes, respectively. To ensure that the activity of RmcA or MorA did not influence the c-di-GMP-dependent transcription of the pel operon by FleQ, the Pel-overproducing strain P. aeruginosa PAO1 $\Delta w s p F$ $\Delta p s / \mathrm{P}_{\mathrm{BAD}}$-pel strain was utilized (36). Coimmunoprecipitation (co-IP) was performed from solubilized, enriched inner membranes of $P$. aeruginosa overexpressing the RmcA-V or MorA-V tagged proteins and the protein components encoded by the pel operon by the addition of L-arabinose to culture media. In each experiment, PeID was detected in the washed resin via Western blot when RmcA-V or MorA-V were supplied as the bait, but not in the untagged control experiment (Fig. 6A). These data suggest that RmcA and MorA may interact with PelD to exert their control over Pel biosynthesis.

While the data gathered by co-IP suggests an interaction between RmcA/MorA and PelD, it does not distinguish between direct interactions or those mediated by other proteins. To validate these findings using a different approach, interactions between RmcA/MorA and PelD were analyzed using bacterial adenylate cyclase two-hybrid (BACTH) assays. In these experiments, the inactive T18 fragment of Bordetella pertussis adenylate cyclase toxin was fused to the $\mathrm{N}$ terminus of RmcA or MorA (T18-RmcA or T18-MorA), while the inactive T25 adenylate cyclase fragment was fused to the $\mathrm{N}$ terminus of PelD (T25-PeID). Interaction between T18-RmcA/T18-MorA and T25-PelD would reconstitute adenylate cyclase enzymatic activity and lead to the production of blue colonies when analyzed in the Escherichia coli BTH101 reporter strain grown on agar medium containing $\mathrm{X}$-Gal (5-bromo-4-chloro-3-indolyl- $\beta$-D-galactopyranoside).

When interactions between T18-RmcA and T25-PeID were examined in the BACTH assay, white colonies were observed, indicative of a negative result (Fig. 6B). Since interactions between RmcA and PeID were identified by co-IP in a P. aeruginosa background where the entire pel operon was overexpressed (Fig. 6A) and PelD directly interacts with both PelE and PelG to form the inner membrane Pel synthase complex regardless of its c-di-GMP binding capability (34), we reasoned that expressing untagged PelE, PelG, or both copolymerase proteins alongside T18-RmcA and T25PelD would better imitate the physiological conditions under which this interaction is presumed to occur. When these modified BACTH experiments were performed, we observed deep blue colonies comparable to the positive control when both PelE and PelG were coexpressed with T18-RmcA and T25-PelD but only very faint blue to white colonies when PelE or PelG were singly coexpressed (Fig. 6B). Similar results were obtained when the modified BACTH experiment was performed with T18-MorA and T25-PelD, where a deep blue colony indicative of a positive result was observed when both untagged PelE and PelG were coexpressed (Fig. 6C). However, unlike with RmcA, a weak-to-moderate positive result was also obtained when only untagged PelE, 
A RmcA RmcA-V MorA MorA-V

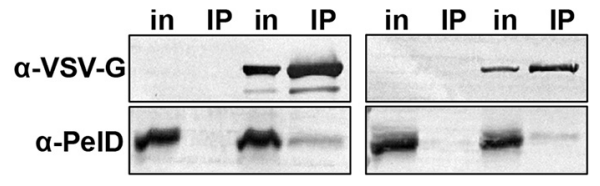

B

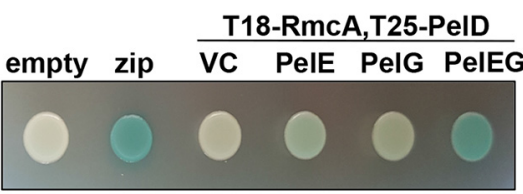

C

T18-MorA, T25-PeID

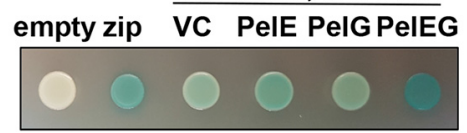

FIG 6 RmcA and MorA physically interact with the Pel biosynthetic machinery. (A) Co-IP from solubilized $P$. aeruginosa PAO1 inner membranes expressing VSV-G-tagged RmcA (RmcA-V, left) or MorA (MorA-V, right) as the bait. The corresponding untagged proteins (RmcA and MorA) were used as a negative binding control. Proteins applied to the $\alpha$-VSV-G co-IP resin (in, input) and the proteins bound to the resin after washing (IP, immunoprecipitated) were analyzed by Western blotting using VSV-G- and PelD-specific antibodies, as indicated. (B) Representative colony images for the analysis of interactions between RmcA, fused at the $\mathrm{N}$ terminus to the T18 domain of Bordetella pertussis adenylate cyclase toxin (T18-RmcA), and PelD, fused to the T25 adenylate cyclase domain at the N terminus (T25-PelD), by BACTH using solid media containing X-Gal as a reporter. The assay was modified through the additional of an empty vector control (VC) for expression of untagged PelE, untagged PelG, or both PelE and PelG (PelEG). A blue colony indicates a positive result in this assay. Empty vectors expressing the T18 or T25 domain alone (empty) were used as a negative control. Fusion of the T18 and T25 domains to a leucine zipper motif (zip) was used as a positive control. (C) Representative colony images for the analysis of interactions between MorA, fused to the T18 domain at the $\mathrm{N}$ terminus (T18-RmcA), and T25-PelD by BACTH assay. Experiments were performed as described for panel $B$. Note that the proteins used in these BACTH assays were derived from $P$. aeruginosa PAO1, which are $99 \%$ identical to the $P$. aeruginosa PA14 proteins.

untagged PelG, or even an empty vector control was present (Fig. 6C). These data collectively show that both RmcA and MorA interact with the Pel biosynthetic machinery, including PelD (34), either directly or indirectly.

\section{DISCUSSION}

Exploiting previous findings from our lab in which a CRISPR-activated strain exhibited a defect in biofilm maintenance (21), we discovered that two PDEs, RmcA and MorA, were essential for maintaining late-stage biofilms. The $\triangle r m c A$ and $\triangle$ morA mutants exhibit phenotypes consistent with the inability to degrade c-di-GMP, specifically, elevated c-di-GMP, increased Pel production, and the ability to initiate a robust biofilm. However, the $\triangle r m c A$ and $\triangle$ morA mutants fail to maintain the biofilm in longterm static assays or when established biofilms are deprived of a carbon source in a microfluidic chamber. In addition, we have shown that the inability to maintain biofilms in these mutant backgrounds is driven by widespread cell death during nutrient limitation. Consistent with the hypothesis that cell death in these mutants is due to an aberrant nutrient limitation response, we showed that the $\Delta$ relA $\Delta$ spoT mutant, which lacks the ability to induce a stringent response, demonstrates a biofilm maintenance defect during nutrient limitation similar to that observed for the $\Delta r m c A$ and $\triangle$ morA mutants.

Taken together, these data suggest a model (Fig. 7) whereby the production of the energetically expensive Pel polysaccharide, required for the initial steps of biofilm formation, is downregulated by RmcA and MorA during biofilm maintenance when nutrient limitation conditions predominate. As such, while the loss of either PDE results in increased EPS levels and enhanced biofilm growth, a boon to these microorganisms in 


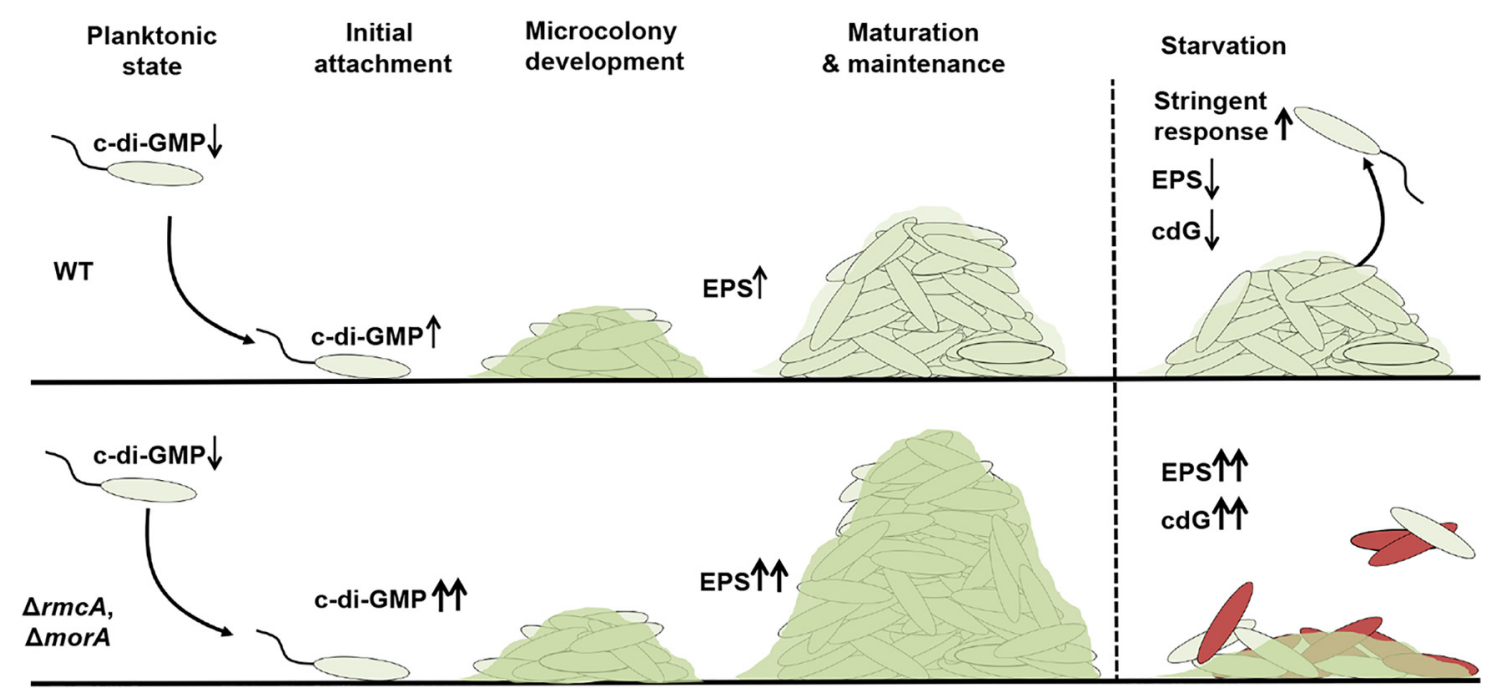

FIG 7 Model for MorA- and RmcA-mediated biofilm maintenance in P. aeruginosa. Typical biofilm development (top panel) involves surface attachment, after which increased c-di-GMP and EPS synthesis mediate microcolony development and increased biofilm biomass. During the maturation and maintenance phase of biofilm development, regulatory changes reflect growth in a nutrient-limited environment and result in a decrease in the production of energetically expensive products like Pel, reduced c-diGMP (cdG) and induction of the stringent response. The loss of RmcA or MorA (bottom panel) results in enhanced c-di-GMP and Pel production and increased biomass in nutrient-rich environments. The $\Delta r m c A$ and $\Delta m o r A$ mutant biofilms are unable to appropriately respond to nutrient limitation, resulting in cell death and loss of biofilm biomass.

resource-rich environments typical of early biofilm formation, it leaves the cells unable to adapt to later nutrient-limited conditions in the context of a mature biofilm.

The mechanisms by which RmcA and MorA are regulated in nutrient-limited conditions remain unknown; however, recent findings in Pseudomonas putida provide a potential signaling framework. Work by Carlos Díaz-Salazar et al. found that RelA and SpoT-dependent synthesis of ( $p$ )ppGpp mediates dispersal during nutrient-limited conditions (37). This group also found that (p)ppGpp increased transcription of the PDE bifA and that a $\triangle$ bifA mutant was defective in starvation-induced biofilm dispersal (37, 38). It is possible that in $P$. aeruginosa RmcA and MorA operate analogously to that of BifA in $P$. putida by acting as effectors of stringent response signaling under nutrientlimited conditions. Unlike BifA in P. putida, RmcA and MorA do not coordinate dispersal in $P$. aeruginosa but rather participate in effectively maintaining the biofilm in the face of nutrient limitation.

It is also possible that the specific impact of RmcA and MorA on late-stage biofilms is due to their expression specifically in mature biofilms. Reported transcriptional analyses suggests that $r m c A$ transcript levels are elevated in $48 \mathrm{~h}$ biofilms versus planktonic culture (39), consistent with this hypothesis. There is no such evidence for enhanced morA transcription in late-stage biofilms, but it is also important to keep in mind that much of c-di-GMP mediated control is via posttranscriptional regulation, as we and many others have shown $(9,13,40)$.

The high levels of c-di-GMP in the $\triangle r m c A$ and $\triangle$ morA mutants may have adverse impacts on the cells in nutritionally limited, mature biofilms. While we do not yet completely understand how the regulation of Pel may contribute to biofilm maintenance, there is strong evidence for the physical interaction of RmcA and MorA with PeID and/ or other elements of the Pel biosynthetic machinery (Fig. 6), suggesting that RmcA and MorA may have a direct role in regulating Pel synthesis. While artificially increasing pel expression alone to high levels does not result in a biofilm maintenance defect, pel gene overexpression combined with enhanced c-di-GMP production (via the wspF mutation) resulted in reduced biomass and increased cell death for these biofilms grown in microfluidics chamber when nutrient-limited. These data are consistent with our findings that overexpression of c-di-GMP alone cannot induce a maintenance 
defect (see Fig. S3) and suggests that it is unregulated production of Pel which makes $P$. aeruginosa biofilms vulnerable in nutrient-limited conditions. Together, with the data presented here indicating that RmcA and MorA physically interact with elements of the Pel machinery, our findings suggest a model whereby RmcA and MorA are components of a Pel regulatory complex required for biofilms to successfully navigate starvation conditions. Finally, we believe that the importance of RmcA- and MorA-mediated regulation of biofilm maintenance extends beyond the in vitro studies we show here, as $P$. aeruginosa strains carrying mutations in $r m c A$ and $\operatorname{mor} A$ genes were the only PDEs to show a defect in a mouse model of catheter infection from among the 15 strains lacking genes encoding proteins with EAL or HD-GYP domains (41).

Further studies are needed to elucidate whether the potential role of Pel in biofilm maintenance is related to the stringent response. It is possible that the inability to appropriately regulate c-di-GMP and EPS production during nutrient limitation impacts (p)ppGpp levels, eventually resulting in extensive cell death and biofilm degradation. Alternatively, the ability to induce a stringent response may be part of a coordinated downregulation of metabolic activity required for the long-term maintenance of a mature biofilm, particularly when carbon/energy sources are limiting. In addition, Pseudomonads appear to have developed catabolic pathways for the utilization of arginine and lactate for "maintenance energy" in mature biofilms $(42,43,58)$, as well as a pathway to downregulate flagellar motility, another early-stage biofilm factor, in mature biofilms (1). Taken together, these data indicate that pseudomonads, and likely other microbes, have active, well-regulated mechanisms necessary to maintain a mature biofilm in the face of changing environmental conditions.

Finally, the apparent role for c-di-GMP-metabolizing enzymes RmcA and MorA later in the biofilm lifestyle suggests the interesting possibility that the plethora of these enzymes in pseudomonads stems from their roles in regulating discrete aspects of the biofilm lifestyle-from formation to maturation to maintenance to dispersal. The finding that the loss of different PDEs would result in different phenotypes may be expected given the varied impacts that these enzymes have on the regulation and timing of c-di-GMP signaling and biofilm formation $(9,13)$. Here, while we assessed all PDE and dual-domain mutants in $P$. aeruginosa in our initial screen, we only observed consistent and significant defects in biofilm maintenance for the $\Delta r m c A$ and $\Delta m o r A$ mutants. Previous work identified a number of DGCs and PDEs apparently required for early biofilm formation, including SadC, RoeA, BifA, and SiaD $(15,16,44)$. In contrast, the PDE DipA has been shown to mediate biofilm dispersion in response to elevated nutrient concentrations and this protein localizes to the cell pole during division, resulting in the asymmetric distribution of c-di-GMP $(18,45)$. Thus, our data are consistent with the hypothesis of stage-specific roles for DGCs/PDEs in the biofilm life cycle.

The network which controls c-di-GMP levels in $P$. aeruginosa is complex. Identified first in $P$. putida, MorA was found to repress motility in swim assays (35). This same work found that the enhanced motility of the morA mutant was not observed in $P$. aeruginosa (35), but previous work from the Hogan and O'Toole labs showed that the $\Delta$ morA mutant exhibited a significant decrease in flagellum-dependent swimming and swarming motility (23). The basis of this difference in phenotypes may be due to the fact that different species of Pseudomonas were used in the two studies. Nevertheless, given the role of motility in early biofilm formation, the observation that MorA contributes to swimming and swarming motility indicates that this PDE also likely contributes to the initiation of biofilm communities.

Insightful work from the Dietrich lab provided evidence that RmcA is activated by phenazine's availability to mediate a decrease in c-di-GMP levels during oxidative stress conditions (24). In this model, RmcA can act as a redox sensor and may behave as a switch to translate this signal into decreased levels of c-di-GMP and EPS. This model for the role of RmcA in the context of the colony biofilm used by Okegbe et al. (24) is largely in agreement with the experimental evidence we have provided, which suggests that RmcA is important for biofilm maintenance. Specifically, it is likely that in 
mature biofilms with elevated biomass, nutrient limitation coincides with oxygen depletion and the production and utilization of phenazines as electron shuttles. Indeed, the direct regulatory signal sensed by RmcA appears to be a change in redox state that is likely secondary to the loss of a catabolizable carbon source (24). Thus, in this environment, we hypothesize that RmcA downregulates the production of energetically expensive Pel EPS and that failure to do so could result in the observed cell death and biofilm maintenance defect. Together, these data suggest that further examination of these enzymes will generate a more nuanced view of the model presented in Fig. 7, wherein specific c-di-GMP metabolizing enzymes work at one or more stages of the biofilm life cycle, with the potential to perform several overlapping functions across these various stages (i.e., biofilm initiation and biofilm maintenance).

\section{MATERIALS AND METHODS}

Strains and media. Strains, plasmids and primers used in this study are listed in Table S1. P. aeruginosa strain UCBPP-PA14 (PA14) was used in all the experiments, unless otherwise indicated, and for the IP studies and the proteins used in the BACTH assay, which used the sequences from the PAO1 strain. $P$. aeruginosa were routinely streaked onto lysogeny broth (LB) plates containing $1.5 \%$ agar prior to overnight culturing in LB liquid cultures at $37^{\circ} \mathrm{C}$. When appropriate, LB was supplemented with $10 \mu \mathrm{g} / \mathrm{ml}$ gentamicin $(\mathrm{Gm})$ and $50 \mu \mathrm{g} / \mathrm{ml}$ kanamycin (Kan). Biofilm medium used in static 96-well crystal violet assays was composed of M63 medium supplemented with $1 \mathrm{mM} \mathrm{MgSO}$ and $0.4 \%$ (wt/vol) L-arginine monochloride and, where indicated, the arginine was replaced by $20 \mathrm{mM}$ pyruvate $(3,46)$. Static and microfluidic-based biofilm assays were performed in KA biofilm medium, a modification of the previously reported $\mathrm{K} 10 \mathrm{~T}$ medium (47) containing $50 \mathrm{mM}$ Tris- $\mathrm{HCl}(\mathrm{pH} 7.4), 0.61 \mathrm{mM} \mathrm{MgSO}_{4}$, and $0.4 \%$ arginine.

Static biofilm assays and quantification. Overnight cultures were inoculated into 96-well U-bottom polystyrene plates (Costar) containing M63-based medium and grown for the specified time at $37^{\circ} \mathrm{C}$ in a hydrated container prior to washing, staining with crystal violet, and then solubilization of the crystal violet stain with $30 \%$ glacial acetic acid $(3,46)$. The extent of the biofilm form was quantified by measuring the extent of biofilm-associated CV solubilized in a spectrophotometer to determine the optical density at $500 \mathrm{~nm}\left(\mathrm{OD}_{550}\right)$.

For biofilms imaged microscopically in static assays, overnight cultures were prepared as described above and inoculated into 12-well dishes containing KA biofilm medium. Glass coverslips were partially submerged in the medium and grown at $37^{\circ} \mathrm{C}$ for the desired length of time prior to removal and imaging with a Nikon Eclipse Ti inverted microscope where a minimum of 10 fields of view were captured. To assess viability of coverslip-grown biofilms, propidium iodide and Syto9 (Molecular Probes Live/Dead BacLight) were gently mixed into the growth medium and biofilms were stained for $1 \mathrm{~h}$ prior to imaging. Pel was visualized with the addition of $10 \mu \mathrm{l} / \mathrm{ml}$ of Pel-specific fluorescein-labeled Wisteria floribunda lectin (Vector Laboratories) to the KA medium at the start of the assay. To assess the concentration of c-diGMP we utilized the $P_{c d r A}$-gfp fusion expressed from a multicopy pMQ72 plasmid (48), which was maintained in overnight cultures supplemented with $\mathrm{Gm}$ prior to inoculation into antibiotic-free KA biofilm medium.

Congo red assay. Strains were grown overnight in $5 \mathrm{ml} \mathrm{LB}$ at $37^{\circ} \mathrm{C}$ and $5 \mu \mathrm{l}$ of an overnight culture was spotted onto plates containing $1.5 \%$ agar, $1 \%$ tryptone, $40 \mu \mathrm{g} / \mathrm{ml} \mathrm{CR}$, and $15 \mu \mathrm{g} / \mathrm{ml}$ Coomassie brilliant blue. The plates were incubated at $37^{\circ} \mathrm{C}$ for $24 \mathrm{~h}$ and imaged after an additional 4 days at room temperature.

Microfluidics. Biofilms were visualized under flow in microfluidics chambers kindly provided by the Nadell laboratory at Dartmouth College. Chambers used polydimethylsiloxane (PDMS) bonded to a cover glass $(1.5 \times 36 \mathrm{~mm} \times 60 \mathrm{~mm}$; Thermo Fisher, Waltham MA) through soft lithography techniques $(49,50)$. Overnight bacterial cultures were centrifuged, resuspended in KA biofilm medium, adjusted to an $\mathrm{OD}_{600}$ of 1 , pipetted into microfluidics chambers, and allowed to attach for $1 \mathrm{~h}$. Tubing (catalog no. 30 Cole-Parmer PTFE) to transport influent and effluent medium was attached first to BD 5-ml syringes containing KA biofilm medium, then to the microfluidics chambers, and then to syringe pumps (Pico Plus Elite; Harvard Apparatus) operating at a flow rate of $0.5 \mu \mathrm{l} / \mathrm{min}$.

Image acquisition and data analysis. All microscopy was acquired using Nikon Elements AR running a Nikon Eclipse Ti inverted microscope equipped with a Hamamatsu ORCA-Flash 4.0 camera and imaged with either a Plan Apochromat $100 \times$ DM Oil or Plan Fluor $40 \times$ DIC M N2 objective. Fast scan mode and $2 \times 2$ binning were used for imaging. All images were collected in a temperature controlled environmental chamber set to $37^{\circ} \mathrm{C}$. Images were processed with background subtraction and signal strength quantified by measuring the mean signal intensity/pixel through the Integrated Density (IntDen) function.

Statistical analysis. Data were analyzed using GraphPad Prism 8. Unless otherwise noted, data are representative of the results from at least three independent experiments. A Student $t$ test of analysis of variance, as indicated, was used to compare results and to assess significance.

Construction of mutant, complementation, and fluorescent strains. Several $P$. aeruginosa strains expressing fluorescent proteins were generated for the imaging studies. $P$. aeruginosa expressing GFP were made through electroporation of a multicopy plasmid pSMC21. The mKO fluorescent protein was 
introduced in single copy on the chromosome at the att:Tn7 site via conjugation from $E$. coli S17- $\lambda$ pir pCN768 (51).

In-frame, unmarked $r m c A$ and morA gene deletions were generated using allelic replacement, as reported previously (52). Construction of gene deletion alleles was performed by amplifying flanking regions of the rmcA and morA open reading frames (ORFs) and joining these flanking regions by splicing-by-overlap extension PCR. The upstream forward and downstream reverse primers were tailed with restriction endonuclease cleavage sequences to enable ligation-dependent cloning of the spliced PCR products. The assembled $\Delta r m c A$ and $\Delta$ morA alleles were ligated into pEX18Gm (53) and the resultant allelic exchange vectors were transformed into E. coli $\mathrm{DH} 5 \alpha$. Plasmids were then isolated from individual colonies and verified by Sanger sequencing using M13F and M13R. Plasmids were conjugated into $P$. aeruginosa PA14 from E. coli, and merodiploids were selected on LB agar containing $10 \mu \mathrm{g} / \mathrm{ml}$ gentamicin. SacB-mediated counter selection was carried out to select for double-crossover mutations on no-salt LB (NSLB) agar containing 15\% (wt/vol) sucrose. Unmarked gene deletions were identified by colony PCR using primers that targeted the outside, flanking regions of the rmcA and morA ORFs. These PCR products were Sanger sequenced using the same primers to confirm the correct deletion. These strains incorporated fluorescent proteins, as described above, as indicated in the text.

The $\Delta r m c A$ and $\Delta$ morA deletion alleles were introduced into $P$. aeruginosa PAO1 $\Delta w s p F \Delta p s / P_{\mathrm{BAD}} p e l$ (36) via biparental mating with the donor strain E. coli SM10 (54). Merodiploids were selected on VogelBonner minimal medium agar containing $30 \mu \mathrm{g} / \mathrm{ml}$ gentamicin. SacB-mediated counter selection was performed to select for double crossover mutations on NSLB agar containing 15\% (wt/vol) sucrose. Unmarked gene deletions were identified by colony PCR using primers that targeted the outside, flanking regions of the $r m c A$ and morA ORFs. These PCR products were Sanger sequenced using the same primers to confirm the correct deletion. These strains incorporated fluorescent proteins, as described above, as indicated in the text.

For gene complementation in P. aeruginosa, pUC18T-miniTn7T-Gm, which allows for single-copy chromosomal insertion of genes (55), was modified to allow for arabinose-dependent expression of complementing genes. The $\mathrm{P}_{\mathrm{BAD}}-$ araC promoter from $\mathrm{pJJH187}$ (56) was amplified using the primer pair miniTn7-pBAD-F and miniTn7-pBAD-R, the latter of which contains flanking sequence encoding Smal, Notl, Pstl, and Ncol sites to generate a multiple cloning site downstream of the araC- $\mathrm{P}_{\mathrm{BAD}}$ promoter. The resulting PCR product was cloned into the Sacl and Hindlll sites of pUC18T-miniTn7T-Gm to generate pUC18T-miniTn7T-Gm-pBAD. The ORF corresponding to rmcA or morA was amplified using primer pairs tailed with restriction endonuclease cleavage sequences to enable ligation-dependent cloning of the PCR products. Upstream primers were also tailed with a synthetic ribosome binding site upstream of the start codon. PCR products were ligated into pUC18T-miniTn7T-Gm-pBAD and the resulting miniTn7 vectors were transformed into $E$. coli $\mathrm{DH} 5 \alpha$ and selected on LB agar containing $10 \mu \mathrm{g} / \mathrm{ml}$ gentamicin and $100 \mu \mathrm{g} / \mathrm{ml}$ carbenicillin. Plasmids were then isolated from individual colonies and verified by Sanger sequencing using the miniTn7-SEQ-F and miniTn7-SEQ-R primers, as well as primers specific to internal portions of each gene, as appropriate. Complemented $P$. aeruginosa strains were generated through incorporation of miniTn7 vectors at the neutral att::Tn7 site on the $P$. aeruginosa chromosome via electroporation of miniTn7 vectors, along with the helper plasmid pTNS2, as previously described (55). Transposon mutants were selected on LB agar containing $30 \mu \mathrm{g} / \mathrm{ml}$ gentamicin.

Incorporation of C-terminal VSV-G tags into p-miniTn7-rmcA and p-miniTn7-morA was performed via PCR with 5'-phosphorylated primer pairs. The forward primer annealed to the stop codon of $r m c A$ or morA plus 15 to $22 \mathrm{bp}$ of downstream vector-encoded sequence. The reverse primer annealed to the coding strand 19 to $21 \mathrm{bp}$ upstream of the rmcA or morA stop codon. The forward and reverse primers contained 5'-overhangs that encoded the last and first halves, respectively, of the VSV-G peptide sequence. The PCR amplified product of these primer pairs was subsequently digested with Dpnl for $1 \mathrm{~h}$ at $37^{\circ} \mathrm{C}$ to remove template DNA, followed by incubation with T4 DNA ligase overnight at room temperature to self-ligate the blunt ends and recircularize the vector. The resulting C-terminally VSV-G-tagged miniTn7 vectors were transformed into E. coli $\mathrm{DH} 5 \alpha$ and selected on LB agar containing $10 \mu \mathrm{g} / \mathrm{ml}$ gentamicin and $100 \mu \mathrm{g} / \mathrm{ml}$ carbenicillin. Plasmids were then isolated from individual colonies and verified by Sanger sequencing as described above.

Coimmunoprecipitation assays. One liter of LB, containing $0.5 \%$ (wt/vol) L-arabinose and $30 \mu \mathrm{g} / \mathrm{ml}$ gentamicin, was inoculated with a $P$. aeruginosa strain carrying a VSV-G-tagged protein and allowed to grow overnight at $37^{\circ} \mathrm{C}$ with shaking at $200 \mathrm{rpm}$. The next day, cells were collected at $5,000 \times g$ for $20 \mathrm{~min}$ at $4^{\circ} \mathrm{C}$. Cell pellets were resuspended in $5 \mathrm{ml}$ of $0.2 \mathrm{M}$ Tris- $\mathrm{HCl}(\mathrm{pH} 8), 1 \mathrm{M}$ sucrose, $1 \mathrm{mM}$ EDTA, and $1 \mathrm{mg} / \mathrm{ml}$ lysozyme. Cells were incubated for $10 \mathrm{~min}$ at room temperature prior to the addition of $20 \mathrm{ml}$ of water, followed by further incubation on ice for $30 \mathrm{~min}$. The resultant solution was centrifuged at $30,000 \times \mathrm{g}$ for $20 \mathrm{~min}$ at $4^{\circ} \mathrm{C}$ to collect spheroplasts. The pellet was then resuspended in $50 \mathrm{ml}$ of $10 \mathrm{mM}$ Tris- $\mathrm{HCl}$ (pH 7.5), $5 \mathrm{mM}$ EDTA, and $1 \mathrm{mM}$ dithiothreitol, and lysed by homogenization using an Emulsiflex-C3 (Avestin, Inc.) at a pressure of 10,000 to $15,000 \mathrm{lb} / \mathrm{in}^{2}$ until the solution appeared translucent. The solution was clarified by centrifugation at $30,000 \times g$ for $20 \mathrm{~min}$ at $4^{\circ} \mathrm{C}$, and the resultant supernatant was further centrifuged at $180,000 \times g$ for $1 \mathrm{~h}$ at $4^{\circ} \mathrm{C}$ to collect the membranes. Membranes were then solubilized in $10 \mathrm{ml}$ of buffer A (50 mM Tris- $\mathrm{HCl}$ [pH 8], $10 \mathrm{mM} \mathrm{MgCl}$, and 2\% [wt/vol] Triton X-100) using a Dounce homogenizer and centrifuged at $90,000 \times g$ for $30 \mathrm{~min}$ at $4^{\circ} \mathrm{C}$ to clarify the solution. $\mathrm{A}$ sample of the solubilized membranes was collected before application to the IP resin as a representative example of the input into the experiment. The IP resin (Sigma anti-VSV-G monoclonal antibody-agarose conjugate) was prepared by mixing $60 \mu \mathrm{l}$ of resin slurry with $10 \mathrm{ml}$ of buffer A, followed by collection of the IP resin by centrifugation at $100 \times g$ for $2 \mathrm{~min}$ at $4^{\circ} \mathrm{C}$ and removal of the supernatant. The solubilized membranes were applied to the washed IP resin and incubated at $4^{\circ} \mathrm{C}$ for $1 \mathrm{~h}$ with agitation. The IP resin 
was then collected by centrifugation at $100 \times g$ for $2 \mathrm{~min}$ at $4^{\circ} \mathrm{C}$, and the supernatant was discarded. The resin was washed five times with $10 \mathrm{ml}$ of buffer $A$ as described above to remove nonspecifically bound protein. The resin was then mixed with $150 \mu \mathrm{l}$ of $2 \times$ Laemmli buffer, boiled at $95^{\circ} \mathrm{C}$ for $10 \mathrm{~min}$, and analyzed by SDS-PAGE followed by Western blotting as described below. As a negative control, the experimental protocol described above was also performed with a $P$. aeruginosa strain carrying the corresponding untagged protein.

Western blot sample analysis. For Western blots, a $0.2-\mu \mathrm{m}$ polyvinylidene difluoride membrane was wetted in methanol and soaked for $5 \mathrm{~min}$ in Western transfer buffer $(25 \mathrm{mM} \mathrm{Tris-HCl}, 150 \mathrm{mM}$ glycine, $20 \%$ [vol $/ \mathrm{vol}$ ] methanol), along with the SDS-PAGE gel to be analyzed. Protein was transferred from the SDS-PAGE gel to the polyvinylidene difluoride membrane by wet blotting $(25 \mathrm{mV}, 2 \mathrm{~h})$. The membrane was briefly washed in Tris-buffered saline (10 mM Tris- $\mathrm{HCl}[\mathrm{pH} 7.5], 150 \mathrm{mM} \mathrm{NaCl})$ containing $0.5 \%$ ( $\mathrm{vol} / \mathrm{vol}$ ) Tween 20 (TBS-T) before blocking in 5\% (wt/vol) skim milk powder in TBS-T for $2 \mathrm{~h}$ at room temperature with gentle agitation. The membrane was briefly washed again in TBS-T before incubation overnight with primary antibody (1:5,000 $\alpha$-PelD polyclonal antibody (34) or 1:75,000 Sigma $\alpha$-VSV-G monoclonal antibody) in TBS-T with $1 \%$ (wt/vol) skim milk powder at $4^{\circ} \mathrm{C}$. The next day, the membrane was washed four times in TBS-T for $5 \mathrm{~min}$ each before incubation for $1 \mathrm{~h}$ with secondary antibody (1:2,000 dilution of Bio-Rad affinity-purified goat $\alpha$-rabbit or goat $\alpha$-mouse IgG conjugated to alkaline phosphatase) in TBS-T with 1\% (wt/vol) skim milk powder. The membrane was then washed four times with TBS-T for 5 min each before development with 5-bromo-4-chloro-3-indolyl phosphate/nitroblue tetrazolium chloride (BioShop ready-to-use BCIP/NBT solution). Developed blots were imaged using a BioRad ChemiDoc imaging system.

Bacterial adenylate cyclase two-hybrid assays. Cloning of rmcA and morA from $P$. aeruginosa PAO1 into the BACTH assay-compatible vector pUT18C and of peID into pKT25 was performed using standard molecular methods. Reverse primers were flanked with a 3 '-stop codon for cloning into pUT18C and pKT25, which encode a N-terminal adenylate cyclase fragment fusion (57). Primer pairs were tailed with restriction endonuclease cleavage sequences to enable ligation-dependent cloning and were used to amplify the corresponding peID, rmc $A$, and morA genes from $P$. aeruginosa PAO1 genomic DNA. PCR products were digested with the appropriate restriction endonucleases and ligated into pUT18C and pKT25, as appropriate. Ligations were transformed into E. coli DH5 $\alpha$ and selected on LB agar containing $50 \mu \mathrm{g} / \mathrm{ml}$ kanamycin for pKT25 clones or $100 \mu \mathrm{g} / \mathrm{ml}$ carbenicillin for pUT18C clones. Plasmids were then isolated from individual colonies and verified by Sanger sequencing using primers specific for pUT18C and pKT25, as well as primers specific to internal segments of $r m c A$ and morA, as appropriate. Positive clones were verified as above.

Combinations of the above T18 and T25 fusion proteins were transformed into the BACTH compatible strain BTH101 (Euromedex) for analysis. For each experiment, $5 \mathrm{ml}$ of LB supplemented with $50 \mu \mathrm{g} /$ $\mathrm{ml}$ kanamycin, $100 \mu \mathrm{g} / \mathrm{ml}$ carbenicillin, and $0.5 \mathrm{mM}$ IPTG (isopropyl- $\beta$-D-thiogalactopyranoside) was inoculated with the appropriate BTH101 strain and grown overnight at $30^{\circ} \mathrm{C}$ with shaking at $200 \mathrm{rpm}$. The next day, $2 \mu \mathrm{l}$ of culture was used to spot inoculate a LB agar plate containing $50 \mu \mathrm{g} / \mathrm{ml} \mathrm{kanamycin}$, $100 \mu \mathrm{g} / \mathrm{ml}$ carbenicillin, $0.5 \mathrm{mM} \mathrm{IPTG}$, and $50 \mu \mathrm{g} / \mathrm{ml} \mathrm{X-Gal}$. The plates were incubated for $24 \mathrm{~h}$ at $30^{\circ} \mathrm{C}$ and subsequently photographed. The vectors pUT18C::zip and pKT25::zip (57) were used as a positive control. Empty pUT18C and pKT25 vectors were used as a negative control.

To generate tag-free expression constructs for the modified BACTH assays, pelE and pelG were amplified from $P$. aeruginosa PAO1 genomic DNA using forward primers that were flanked with a synthetic ribosome binding site and reverse primers flanked with a 3 '-stop codon. PCR products were subsequently digested with EcoRI and HindIII and ligated into the arabinose-inducible expression vector pBADGr (10 clones with positive inserts were verified by Sanger sequencing using the BADGr-SEQ-F and BADGr-SEQ-R primers). The vector expressing both pelE and pelG was generated by amplifying the pelE and pelG ORFs from $P$. aeruginosa PAO1 genomic DNA. The intervening pelF ORF from the pel operon was excluded by joining the upstream peIE and downstream pelG ORFs via the splicing-by-overlap extension PCR method, as described above for generation of chromosomal deletion alleles, to generate a single polycistronic strand. All assays with untagged constructs were performed as described above, with the addition of $10 \mu \mathrm{g} / \mathrm{ml}$ gentamicin and $0.5 \%$ (wt/vol) arabinose to all growth media for, respectively, maintenance of and expression from pBADGr. Empty pBADGr was used as a vector control.

\section{SUPPLEMENTAL MATERIAL}

Supplemental material is available online only.

FIG S1, PDF file, $0.1 \mathrm{MB}$.

FIG S2, PDF file, $0.1 \mathrm{MB}$.

FIG S3, PDF file, $0.1 \mathrm{MB}$.

FIG S4, PDF file, $0.1 \mathrm{MB}$.

FIG S5, PDF file, $0.1 \mathrm{MB}$.

FIG S6, PDF file, $0.1 \mathrm{MB}$.

FIG S7, PDF file, $0.1 \mathrm{MB}$.

FIG S8, PDF file, $0.1 \mathrm{MB}$.

FIG S9, PDF file, $0.3 \mathrm{MB}$.

TABLE S1, PDF file, $0.1 \mathrm{MB}$. 


\section{ACKNOWLEDGMENTS}

We thank C. Nadell for providing the fluorescent protein constructs and help with preparing the microfluidic chambers. We thank A. Collins and G. Heussler for constructive feedback throughout the course of this research.

This study was supported by NIH grant R37 Al83256-06 to G.A.O. and the Canadian Institutes of Health Research (CIHR) MOP 43998 and FDN154327 to P.L.H. P.L.H. is the recipient of a Tier I Canada Research Chair. G.B.W. was supported in part by a Canada Graduate Scholarship from the Natural Sciences and Engineering Research Council of Canada (NSERC) and a graduate scholarship from Cystic Fibrosis Canada.

\section{REFERENCES}

1. Sauer K, Camper AK, Ehrlich GD, Costerton JW, Davies DG. 2002. Pseudomonas aeruginosa displays multiple phenotypes during development as a biofilm. J Bacteriol 184:1140-1154. https://doi.org/10.1128/jb.184.4.1140 $-1154.2002$.

2. Toutain CM, Caizza NC, Zegans ME, O'Toole GA. 2007. Roles for flagellar stators in biofilm formation by Pseudomonas aeruginosa. Res Microbiol 158:471-477. https://doi.org/10.1016/j.resmic.2007.04.001.

3. O'Toole GA, Kolter R. 1998. Flagellar and twitching motility are necessary for Pseudomonas aeruginosa biofilm development. Mol Microbiol 30:295-304. https://doi.org/10.1046/j.1365-2958.1998.01062.x.

4. Ghafoor A, Hay ID, Rehm BHA. 2011. Role of exopolysaccharides in Pseudomonas aeruginosa biofilm formation and architecture. Appl Environ Microbiol 77:5238-5246. https://doi.org/10.1128/AEM.00637-11.

5. Colvin KM, Gordon VD, Murakami K, Borlee BR, Wozniak DJ, Wong GCL, Parsek MR. 2011. The Pel polysaccharide can serve a structural and protective role in the biofilm matrix of Pseudomonas aeruginosa. PLoS Pathog 7:e1001264. https://doi.org/10.1371/journal.ppat.1001264.

6. Flemming HC, Wingender J. 2001. Relevance of microbial extracellular polymeric substances (EPSs). I. Structural and ecological aspects. Water Sci Technol 43:1-8. https://doi.org/10.2166/wst.2001.0326.

7. Jenal U, Reinders A, Lori C. 2017. Cyclic di-GMP: second messenger extraordinaire. Nat Rev Microbiol 15:271-284. https://doi.org/10.1038/nrmicro .2016.190.

8. Simm R, Morr M, Kader A, Nimtz M, Römling U. 2004. GGDEF and EAL domains inversely regulate cyclic di-GMP levels and transition from sessibility to motility. Mol Microbiol 53:1123-1134. https://doi.org/10.1111/j .1365-2958.2004.04206.x.

9. Valentini M, Filloux A. 2019. Multiple roles of c-di-GMP signaling in bacterial pathogenesis. Annu Rev Microbiol 73:387-406. https://doi.org/10 .1146/annurev-micro-020518-115555.

10. Hickman JW, Harwood CS. 2008. Identification of FleQ from Pseudomonas aeruginosa as a c-di-GMP-responsive transcription factor. Mol Microbiol 69:376-389. https://doi.org/10.1111/j.1365-2958.2008.06281.x.

11. Stelitano V, Giardina G, Paiardini A, Castiglione N, Cutruzzolà F, Rinaldo S. 2013. C-di-GMP hydrolysis by Pseudomonas aeruginosa HD-GYP phosphodiesterases: analysis of the reaction mechanism and novel roles for $\mathrm{pGpG}$. PLoS One 8(9):e74920. https://doi.org/10.1371/journal.pone.0074920.

12. Kulasakara $H$, Lee V, Brencic A, Liberati N, Urbach J, Miyata S, Lee DG, Neely AN, Hyodo M, Hayakawa Y, Ausubel FM, Lory S. 2006. Analysis of Pseudomonas aeruginosa diguanylate cyclases and phosphodiesterases reveals a role for bis- $\left(3^{\prime}-5^{\prime}\right)$-cyclic-GMP in virulence. Proc Natl Acad Sci U S A 103:2839-2844. https://doi.org/10.1073/pnas.0511090103.

13. Ha D-G, O'Toole GA. 2015. c-di-GMP and its effects on biofilm formation and dispersion: a Pseudomonas aeruginosa review. Microbiol Spectr 3:1-12.

14. Romling U, Galperin MY, Gomelsky M. 2013. Cyclic di-GMP: the first 25 Years of a universal bacterial second messenger. Microbiol Mol Biol Rev 77:1-52. https://doi.org/10.1128/MMBR.00043-12.

15. Merritt JH, Brothers KM, Kuchma SL, O'Toole GA. 2007. SadC reciprocally influences biofilm formation and swarming motility via modulation of exopolysaccharide production and flagellar function. J Bacteriol 189:8154-8164. https://doi.org/10.1128/JB.00585-07.

16. Kuchma SL, Brothers KM, Merritt JH, Liberati NT, Ausubel FM, O'Toole GA. 2007. BifA, a cyclic-di-GMP phosphodiesterase, inversely regulates biofilm formation and swarming motility by Pseudomonas aeruginosa PA14. J Bacteriol 189:8165-8178. https://doi.org/10.1128/JB.00586-07.

17. Hickman JW, Tifrea DF, Harwood CS. 2005. A chemosensory system that regulates biofilm formation through modulation of cyclic diguanylate levels. Proc Natl Acad Sci U S A 102:14422-14427. https://doi.org/10 $.1073 /$ pnas.0507170102.

18. Roy AB, Petrova OE, Sauer K. 2012. The phosphodiesterase DipA (PA5017) is essential for Pseudomonas aeruginosa biofilm dispersion. J Bacteriol 194:2904-2915. https://doi.org/10.1128/JB.05346-11.

19. Li Y, Heine S, Entian M, Sauer K, Frankenberg-Dinkel N. 2013. NO-induced biofilm dispersion in Pseudomonas aeruginosa is mediated by an MHYT domain-coupled phosphodiesterase. J Bacteriol 195:3531-3542. https:// doi.org/10.1128/JB.01156-12.

20. Southey-Pillig CJ, Davies DG, Sauer K. 2005. Characterization of temporal protein production in Pseudomonas aeruginosa biofilms. J Bacteriol 187:8114-8126. https://doi.org/10.1128/JB.187.23.8114-8126.2005.

21. Heussler GE, Cady KC, Koeppen K, Bhuju S, Stanton BA, O’Toole GA. 2015. Clustered regularly interspaced short palindromic repeat-dependent, biofilm-specific death of Pseudomonas aeruginosa mediated by increased expression of phage-related genes. mBio 6:e00129-15. https://doi.org/10 $.1128 / \mathrm{mBio} .00129-15$.

22. Schultz J, Milpetz F, Bork P, Ponting CP. 1998. SMART, a simple modular architecture research tool: identification of signaling domains. Proc Natl Acad Sci U S A 95:5857-5864. https://doi.org/10.1073/pnas.95.11.5857.

23. Ha D-G, Richman ME, O'Toole GA. 2014. Deletion mutant library for investigation of functional outputs of cyclic diguanylate metabolism in Pseudomonas aeruginosa PA14. Appl Environ Microbiol 80:3384-3393. https:// doi.org/10.1128/AEM.00299-14.

24. Okegbe C, Fields BL, Cole SJ, Beierschmitt C, Morgan CJ, Price-Whelan A, Stewart RC, Lee VT, Dietrich LEP. 2017. Electron-shuttling antibiotics structure bacterial communities by modulating cellular levels of c-diGMP. Proc Natl Acad Sci U S A 114:E5236-E5245. https://doi.org/10.1073/ pnas. 1700264114.

25. Phippen CW, Mikolajek H, Schlaefli HG, Keevil CW, Webb JS, Tews I. 2014. Formation and dimerization of the phosphodiesterase active site of the Pseudomonas aeruginosa MorA, a bi-functional c-di-GMP regulator. FEBS Lett 588:4631-4636. https://doi.org/10.1016/j.febslet.2014.11.002.

26. Bloemberg GV, O'Toole GA, Lugtenberg BJ, Kolter R. 1997. Green fluorescent protein as a marker for Pseudomonas spp. Appl Environ Microbiol 63:4543-4551. https://doi.org/10.1128/AEM.63.11.4543-4551.1997.

27. Mah T-F, Pitts B, Pellock B, Walker GC, Stewart PS, O'Toole GA. 2003. A genetic basis for Pseudomonas aeruginosa biofilm antibiotic resistance. Nature 426:306-310. https://doi.org/10.1038/nature02122.

28. Jennings LK, Storek KM, Ledvina HE, Coulon C, Marmont LS, Sadovskaya I, Secor PR, Tseng BS, Scian M, Filloux A, Wozniak DJ, Howell PL, Parsek MR. 2015. Pel is a cationic exopolysaccharide that cross-links extracellular DNA in the Pseudomonas aeruginosa biofilm matrix. Proc Natl Acad Sci U S A 112:11353-11358. https://doi.org/10.1073/pnas.1503058112.

29. Merritt JH, Kadouri DE, O'Toole GA. 2005. Growing and analyzing static biofilms. Curr Protoc Microbiol 22:1-17.

30. Friedman L, Kolter R. 2004. Genes involved in matrix formation in Pseudomonas aeruginosa PA14 biofilms. Mol Microbiol 51:675-690. https://doi .org/10.1046/j.1365-2958.2003.03877.x.

31. Baraquet C, Murakami K, Parsek MR, Harwood CS. 2012. The FleQ protein from Pseudomonas aeruginosa functions as both a repressor and an activator to control gene expression from the Pel operon promoter in response to c-di-GMP. Nucleic Acids Res 40:7207-7218. https://doi.org/10 .1093/nar/gks384.

32. Lee VT, Matewish JM, Kessler JL, Hyodo M, Hayakawa Y, Lory S. 2007. A cyclic-di-GMP receptor required for bacterial exopolysaccharide production. Mol Microbiol 65:1474-1484. https://doi.org/10.1111/j.1365-2958.2007 .05879.x. 
33. Dahlstrom KM, O'Toole GA. 2017. A symphony of cyclases: specificity in diguanylate cyclase signaling. Annu Rev Microbiol 71:179-195. https:// doi.org/10.1146/annurev-micro-090816-093325.

34. Whitfield GB, Marmont LS, Ostaszewski A, Rich JD, Whitney JC, Parsek MR, Harrison JJ, Howell PL. 2020. Pel polysaccharide biosynthesis requires an inner membrane complex comprised of PelD, PelE, PelF, and PelG. J Bacteriol 202:e00684-19. https://doi.org/10.1128/JB.00684-19.

35. Choy WK, Zhou L, Syn CKC, Zhang LH, Swarup S. 2004. MorA defines a new class of regulators affecting flagellar development and biofilm formation in diverse Pseudomonas species. J Bacteriol 186:7221-7228. https://doi.org/10.1128/JB.186.21.7221-7228.2004.

36. Colvin KM, Alnabelseya N, Baker P, Whitney JC, Lynne Howell P, Parsek MR. 2013. PelA deacetylase activity is required for Pel polysaccharide synthesis in Pseudomonas aeruginosa. J Bacteriol 195:2329-2339. https://doi .org/10.1128/JB.02150-12.

37. Díaz-Salazar $C$, Calero $P$, Espinosa-Portero $R$, Jiménez-Fernández $A$, Wirebrand L, Velasco-Domínguez MG, López-Sánchez A, Shingler V, Govantes F. 2017. The stringent response promotes biofilm dispersal in Pseudomonas putida. Sci Rep 7:1-13. https://doi.org/10.1038/s41598-017 $-18518-0$.

38. Jimenez-Fernandez A, Lopez-Sanchez A, Calero P, Govantes F. 2015. The c-di-GMP phosphodiesterase BifA regulates biofilm development in Pseudomonas putida. Environ Microbiol Rep 7:78-84. https://doi.org/10.1111/ 1758-2229.12153.

39. Dötsch A, Eckweiler D, Schniederjans M, Zimmermann A, Jensen V, Scharfe M, Geffers R, Häussler S. 2012. The Pseudomonas aeruginosa transcriptome in planktonic cultures and static biofilms using RNA sequencing. PLoS One 7:e31092. https://doi.org/10.1371/journal.pone.0031092.

40. Dahlstrom KM, Collins AJ, Doing G, Taroni JN, Gauvin TJ, Greene CS, Hogan DA, O'Toole GA. 2018. A multimodal strategy used by a large c-di-GMP network. J Bacteriol 200:e00703-17. https://doi.org/10.1128/JB.00703-17.

41. Cole SJ, Lee VT. 2016. Cyclic di-GMP signaling contributes to Pseudomonas aeruginosa-mediated catheter-associated urinary tract infection. J Bacteriol 198:91-97. https://doi.org/10.1128/JB.00410-15.

42. Bernier SP, Ha D-G, Khan W, Merritt JH, O'Toole GA. 2011. Modulation of Pseudomonas aeruginosa surface-associated group behaviors by individual amino acids through c-di-GMP signaling. Res Microbiol 162:680-688. https://doi.org/10.1016/j.resmic.2011.04.014.

43. Lin YC, Cornell WC, Jo J, Price-Whelan A, Dietrich LEP. 2018. The Pseudomonas aeruginosa complement of lactate dehydrogenases enables use of D- and L-lactate and metabolic cross-feeding. mBio 9:e00961-18. https:// doi.org/10.1128/mBio.00961-18.

44. Klebensberger J, Birkenmaier A, Geffers R, Kjelleberg S, Philipp B. 2009. $\mathrm{Sia} A$ and $\mathrm{SiaD}$ are essential for inducing autoaggregation as a specific response to detergent stress in Pseudomonas aeruginosa. Environ Microbiol 11:3073-3086. https://doi.org/10.1111/j.1462-2920.2009.02012.x.

45. Kulasekara BR, Kamischke C, Kulasekara HD, Christen M, Wiggins PA, Miller SI. 2013. c-di-GMP heterogeneity is generated by the chemotaxis machinery to regulate flagellar motility. Elife 2:eLife.01402. https://doi .org/10.7554/eLife.01402.
46. Cady KC, O'Toole GA. 2011. Non-identity-mediated CRISPR-bacteriophage interaction mediated via the Csy and Cas3 proteins. J Bacteriol 193:3433-3445. https://doi.org/10.1128/JB.01411-10.

47. Monds RD, Newell PD, Schwartzman JA, O'Toole GA. 2006. Conservation of the Pho regulon in $P$. fluorescens Pfo-1. Appl Environ Microbiol 72:1910-1924. https://doi.org/10.1128/AEM.72.3.1910-1924.2006.

48. Shanks RMQ, Caiazza NC, Hinsa SM, Toutain CM, O'Toole GA. 2006. Saccharomyces cerevisiae-based molecular tool kit for manipulation of genes from Gram-negative bacteria. Appl Environ Microbiol 72:5027-5036. https://doi.org/10.1128/AEM.00682-06.

49. Sia SK, Whitesides GM. 2003. Microfluidic devices fabricated in poly(dimethylsiloxane) for biological studies. Electrophoresis 24:3563-3576. https:// doi.org/10.1002/elps.200305584.

50. Weibel DB, DiLuzio WR, Whitesides GM. 2007. Microfabrication meets microbiology. Nat Rev Microbiol 5:209-218. https://doi.org/10.1038/nrmicro1616.

51. Wucher BR, Bartlett TM, Hoyos M, Papenfort K, Persat A, Nadell CD. 2019. Vibrio cholerae filamentation promotes chitin surface attachment at the expense of competition in biofilms. Proc Natl Acad Sci U S A 116:14216-14221. https://doi .org/10.1073/pnas.1819016116.

52. Hmelo LR, Borlee BR, Almblad H, Love ME, Randall TE, Tseng BS, Lin C, Irie Y, Storek KM, Yang JJ, Siehnel RJ, Howell PL, Singh PK, Tolker-Nielsen T, Parsek MR, Schweizer HP, Harrison JJ. 2015. Precision-engineering the Pseudomonas aeruginosa genome with two-step allelic exchange. Nat Protoc 10:1820-1841. https://doi.org/10.1038/nprot.2015.115.

53. Hoang TT, Karkhoff-Schweizer RR, Kutchma AJ, Schweizer HP. 1998. A broad-host-range F1p-FRT recombination system for site-specific excision of chromosomally-located DNA sequences: application for isolation of unmarked Pseudomonas aeruginosa mutants. Gene 212:77-86. https://doi .org/10.1016/s0378-1119(98)00130-9.

54. de Lorenzo V, Timmis KN. 1994. Analysis and construction of stable phenotypes in gram-negative bacteria with Tn5- and Tn10-derived minitransposons. Methods Enzymol 235:386-405. https://doi.org/10.1016/0076 -6879(94)35157-0.

55. Choi KH, Gaynor JB, White KG, Lopez C, Bosio CM, Karkhoff-Schweizer RAR, Schweizer HP. 2005. A Tn7-based broad-range bacterial cloning and expression system. Nat Methods 2:443-448. https://doi.org/10.1038/nmeth765.

56. Almblad H, Harrison JJ, Rybtke M, Groizeleau J, Givskov M, Parsek MR, Tolker-Nielsen T. 2015. The cyclic AMP-Vfr signaling pathway in Pseudomonas aeruginosa is inhibited by cyclic di-GMP. J Bacteriol 197:2190-2200. https://doi.org/10.1128/JB.00193-15.

57. Karimova G, Ullmann A, Ladant D. 2001. Protein-protein interaction between Bacillus stearothermophilus tyrosyl-tRNA synthetase subdomains revealed by a bacterial two-hybrid system. J Mol Microbiol Biotechnol 3:73-82.

58. Petrova OE, Schurr JR, Schurr MJ, Sauer K. 2012. Microcolony formation by the opportunistic pathogen Pseudomonas aeruginosa requires pyruvate and pyruvate fermentation. Mol Microbiol 86:819-835. https://doi .org/10.1111/mmi.12018. 